\title{
UV Radiation Fields Produced by Young Embedded Star Clusters
}

\author{
Marco Fatuzzo ${ }^{1}$ and Fred C. Adams ${ }^{2,3}$ \\ ${ }^{1}$ Physics Department, Xavier University, Cincinnati, OH 45207 \\ ${ }^{2}$ Michigan Center for Theoretical Physics, University of Michigan \\ Physics Department, Ann Arbor, MI 48109 \\ ${ }^{3}$ Astronomy Department, University of Michigan, Ann Arbor, MI 48109 \\ fatuzzo@xavier.edu, fca@umich.edu
}

\begin{abstract}
A large fraction of stars form within young embedded clusters, and these environments produce a substantial ultraviolet (UV) background radiation field, which can provide feedback on the star formation process. To assess the possible effects of young stellar clusters on the formation of their constituent stars and planets, this paper constructs the expected radiation fields produced by these clusters. We include both the observed distribution of cluster sizes $N$ in the solar neighborhood and an extended distribution that includes clusters with larger $N$. The paper presents distributions of the FUV and EUV luminosities for clusters with given stellar membership $N$, distributions of FUV and EUV luminosity convolved over the expected distribution of cluster sizes $N$, and the corresponding distributions of FUV and EUV fluxes. These flux distributions are calculated both with and without the effects of extinction. Finally, we consider the effects of variations in the stellar initial mass function on these radiation fields. Taken together, these results specify the distributions of radiation environments that forming solar systems are expected to experience.
\end{abstract}

Subject headings: stars: formation - planets: formation - open clusters

\section{INTRODUCTION}

Although a robust paradigm for star formation within giant molecular cloud complexes now exists (Shu et al. 1987), most stars are thought to be born within some type of cluster environment (e.g., Lada \& Lada 2003; Porras et al. 2003). Furthermore, the distribution 
of cluster sizes (given here in terms of stellar membership $N$ ) remains uncertain, and the influence of the cluster environment on forming stars and planets is not completely understood. One important way in which the cluster setting can influence the formation of additional cluster members, and especially their accompanying planetary systems, is through the radiation fields provided by the background environment. This radiation can potentially drive a number of significant processes, including [1] the heating of starless cores, leading to evaporation and the loss of star forming potential (e.g., Gorti \& Hollenbach 2002), [2] the evaporation of circumstellar disks, leading to loss of planet forming potential (e.g., Shu et al. 1993, Hollenbach et al. 1994, Störzer \& Hollenbach 1999, Adams et al. 2004), [3] ionization within starless cores, leading to greater coupling between the magnetic fields and gas (e.g., Shu 1992), thereby acting to suppress continued star formation, and [4] ionization of circumstellar disks, which helps maintain the magneto-rotational instability (MRI), which in turn helps drive disk accretion (e.g., Balbus \& Hawley 1991). For applications to circumstellar disks, note that the background radiation from the cluster environment often dominates that produced by the central star (e.g., Hollenbach et al. 2000; Adams \& Myers 2001); this claim is substantiated and quantified by the results of this paper.

The goal of this paper is to provide a systematic construction of the distributions of the expected radiation fields in clusters, including UV luminosities and fluxes, in both the EUV and FUV radiation bands (these are defined in $\S 2$ ). With these distributions of luminosities and fluxes determined, one can then assess the importance of the cluster background environment for star and planet formation, through the channels outlined above (and others). Although a complete assessment of this type has not been done, the importance of radiation fields in clusters has been emphasized in previous work. A study of the EUV radiation fields and their potentially harmful effects on planet formation has been carried out (Armitage 2000). An analogous treatment of the FUV radiation fields for the clusters in the solar neighborhood has also been done (Adams et al. 2006; hereafter APFM). Finally, Parravano et al. (2003) have reconstructed the UV radiation fields in the local interstellar medium, where the ultimate source of the UV radiation is star forming regions.

This paper is organized as follows. In $\S 2$ we outline the basic approach, including specification of the cluster sample, assumptions about the gas content, and our characterization of the stellar IMF. We then present the distributions of EUV and FUV luminosities in $\S 3$, where these distributions are constructed both for clusters of a given size $N$ and for the entire ensemble of cluster sizes. In $\S 4$, we present the corresponding distributions of EUV and FUV flux, which requires the additional specification of the distribution of radial positions within the cluster. This section also discusses the effects of extinction and the effects of averaging over stellar orbits on the resulting distributions. We conclude in $\S 5$ with a summary of our findings and a discussion of potential applications. 


\section{FORMULATION}

In this section, we specify the input parameters used to produce the resulting distributions of radiation fields. We must specify the distribution of cluster membership sizes $N$, the cluster radii as a function of $N$, the stellar initial mass function (IMF), and the mass-luminosity relationship for massive stars. We defer a discussion of the gas content of clusters, and our treatment of extinction, until $\S 4$ where we consider the flux distributions. Throughout this paper, we often present results as a function of cluster size $N$. In this context, we consider the cluster to have a stellar membership of $N$ primaries and we ignore binarity. We also present results for both FUV radiation, which is defined to have photon energies in the range $6 \mathrm{eV} \leq h \nu \leq 13.6 \mathrm{eV}$, and EUV radiation, where $h \nu \geq 13.6 \mathrm{eV}$. To define nomenclature: We use the general subscript ' $U V$ ' to denote either of these ultraviolet radiation bands, and the explicit designation ' $E U V$ ' or ' $F U V$ ' to denote one of the two particular UV radiation bands.

For the distribution of cluster sizes (stellar membership $N$ ), we use two standard choices. The first is the observed distribution of cluster sizes in the stellar neighborhood. In this case, both Lada \& Lada (2003) and Porras et al. (2003) have produced distributions of observed clusters out to $2 \mathrm{kpc}(1 \mathrm{kpc})$ and down to cluster sizes of 35 (30), respectively. These observational compilations show that the number of stars born in clusters of size $N$ is evenly distributed logarithmically over the range of clusters seen in the solar neighborhood, i.e., from $N=30$ to 2000 . For the sake of definiteness, we use the actual data from the Lada \& Lada (2003) compilation as our first standard distribution. While this catalog is not complete, it is likely representative of the basic statistical properties of embedded clusters within $2 \mathrm{kpc}$. For our second standard distribution, we assume that this general trend continues up to larger cluster sizes, i.e., that star formation takes place in clusters with an even logarithmic distribution extending up to $N=10^{5}$ stars. Both the Lada/Lada distribution and the extended distribution are shown in Figure 1.

The radial sizes of observed clusters follow a well-defined law of the form

$$
R_{\mathrm{c}}(N)=R_{0}\left(\frac{N}{300}\right)^{1 / 2},
$$

where the scale $R_{0} \approx 1$ pc (see Fig. 2 of APFM, which uses the data from Lada \& Lada 2003, and Carpenter 2000). We use this empirically determined law to specify cluster radii throughout this paper, including for the extrapolation of the cluster distribution described above.

The stellar IMF has a power-law form, with a nearly universal slope, for masses $M_{*} \gtrsim 1 M_{\odot}$ (starting with Salpeter 1955) and a lognormal form below this value. Since the total lumi- 


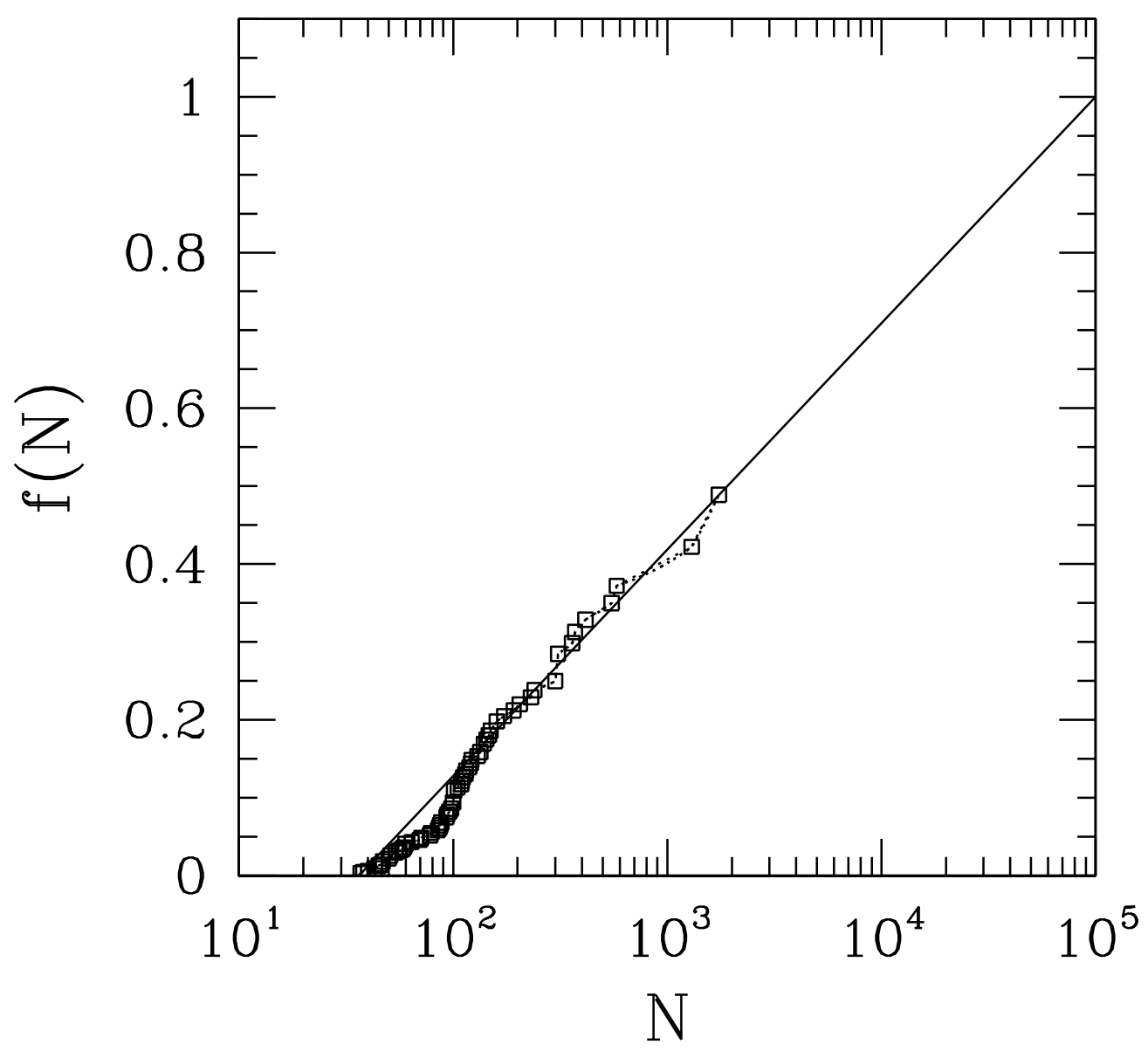

Fig. 1.- Cumulative distribution showing the fraction of stars born in clusters of stellar membership $N$ as a function of $N$. The data points with the dotted curve show the observed distribution in the solar neighborhood (as compiled in Lada \& Lada 2003). The solid line shows an extended distribution for which the same trend - roughly equal number of stars born in each decade of cluster size - extends up to $N=10^{5}$. Note that the scale for $f(N)$ is given for the extended distribution; for the solar neighborhood, $f(N)=1$ for $N=1740$. 
nosity of a star scales roughly as $m^{3}$ for stars with mass less than $\sim 10 M_{\odot}$ and the IMF has a slope of $\sim 2.35$, the luminosity distribution as a function of stellar mass scales as $\sim m^{0.7}$ for intermediate mass stars. Of course, low mass stars fall within the lognormal part of the IMF and have spectra that peak well below the UV band. As a result, we can ignore the contribution of all stars smaller than $1 M_{\odot}$ to a reasonable approximation (as quantified in Fig. 2 below). To specify the initial mass function in this context, we only need to correctly account for the fraction $\mathcal{F}_{1}$ of stars with $M_{*}>1 M_{\odot}$ and the slope $\Gamma$ at high stellar masses. We thus assume that the stellar IMF has a power-law form for mass $M_{*}>1 M_{\odot}$ with index Г, i.e.,

$$
\frac{d N_{\star}}{d m}=A m^{-\Gamma}=\mathcal{F}_{1}(\Gamma-1) m^{-\Gamma}
$$

where $m$ is the mass in units of solar masses and where the slope $\Gamma=2.35$ for the classic form of Salpeter (1955). Although this slope is remarkably consistent over a wide variety of regions (Massey 2003 and references therein), we can account for possible variations in the IMF by allowing the index $\Gamma$ to vary. In the second equality, we have normalized the distribution according to the convention

$$
\int_{1}^{m_{\max }} \frac{d N_{\star}}{d m} d m=\mathcal{F}_{1},
$$

so that $\mathcal{F}_{1}$ is defined to be the fraction of the stellar population with masses larger than 1 $M_{\odot}$. For a typical stellar mass function (e.g., that advocated by Adams \& Fatuzzo 1996), the fraction $\mathcal{F}_{1} \approx 0.12$. Notice also that we assume that the IMF does not extend up to arbitrarily high stellar masses, but rather is truncated at a maximum mass $m_{\max }$. In this context, the IMF is thus determined by the parameter set $\left(\mathcal{F}_{1}, \Gamma, m_{\max }\right)$. In this paper, we fix $\mathcal{F}_{1}=0.12$ for all cases and vary the other two parameters such that $\left(\Gamma, m_{\max }\right)=(2.35$, $100),(2.1,100),(2.5,100)$, and $(2.35,120)$. We adopt the first of these choices of IMF parameters as our standard case, but explore the effects of varying the IMF in $\S 4$.

With the IMF specified, we need to determine the relationship between stellar mass and UV luminosity. Toward that end, we use stellar models calculated by Maeder and collaborators (e.g., Maeder \& Meynet 1987; Schaller et al. 1992). Specifically, this previous work provides a grid of stellar models as a function of both mass and age. In this setting we use the zero age models to specify the stellar luminosity and effective temperature. We then follow the procedure of Armitage (2000) to determine the luminosity in the EUV and FUV bands (note that departures from blackbody spectra are important in this regime). Notice also that the UV radiation is dominated by the largest stars, which reach the mainsequence rapidly, so zero-age main sequence models are appropriate for the construction of these luminosity distributions (i.e., we need not consider pre-main-sequence evolution). Additional stellar evolution results in two competing effects for the total cluster luminosity: 


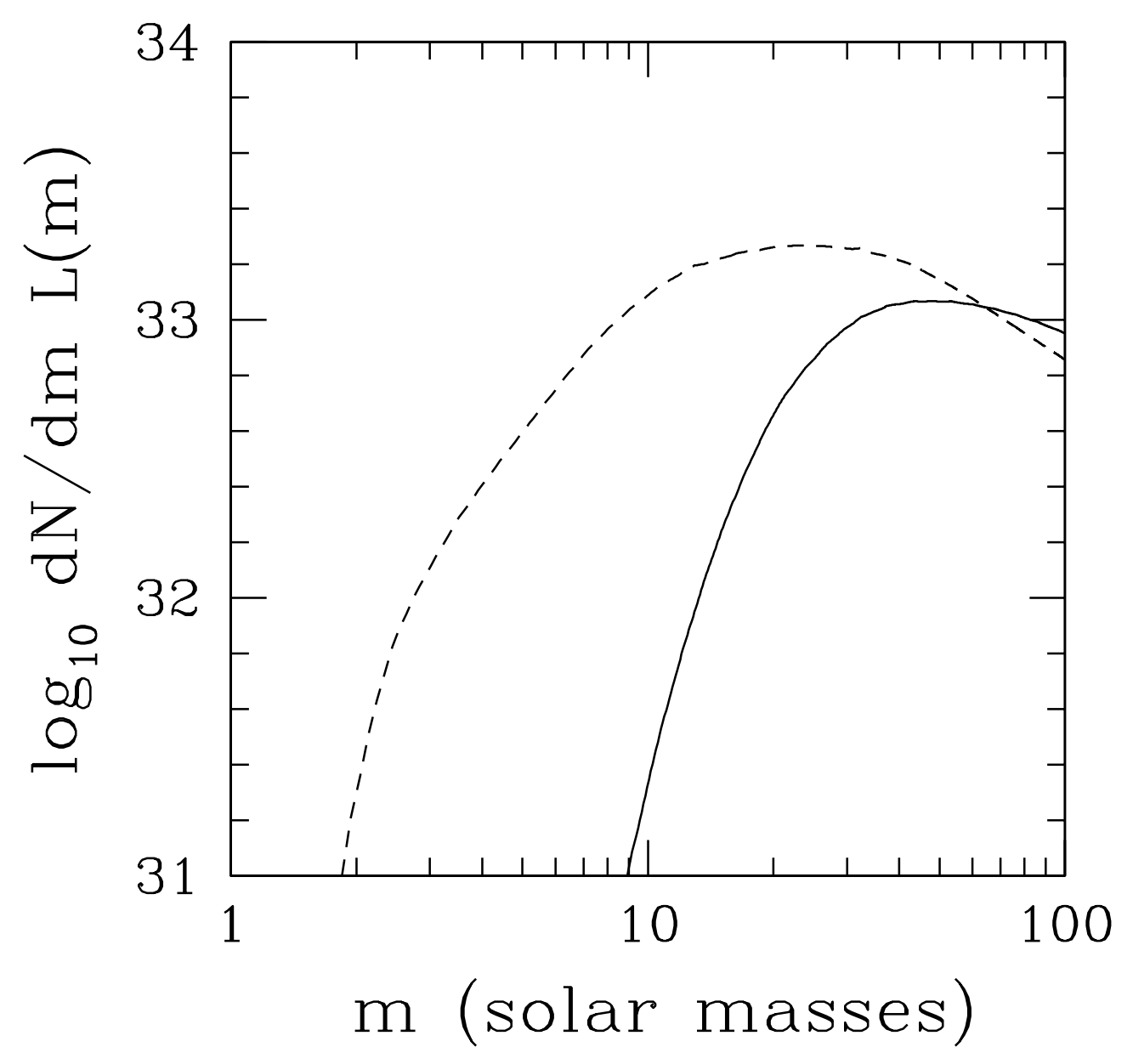

Fig. 2.- Distribution of UV luminosity over the range of stellar masses. The solid line shows relative amount of EUV luminosity produced by stars of varying mass, plotted as a function of mass. The dashed line shows the analogous distribution for FUV luminosity. The vertical axis gives the distribution in units of $\operatorname{erg~s}^{-1} M_{\odot}^{-1}$. The IMF is normalized as in equation (3). 
All stars get brighter as they age, but the most massive stars (with $m \sim 100$ ) have relatively short lifetimes. Adopting zero-age main sequence values for the luminosity, as done here, thus yields one representative outcome for a given cluster. The next level of complication, not done here, would be to construct distributions of UV luminosity and flux as a function of cluster age.

Figure 2 shows the range of stellar masses that dominates the production of UV radiation for both the EUV and FUV bands. The dashed curve shows the standard IMF multiplied by the FUV luminosity, plotted as a function of stellar mass. The distribution shows a broad peak near $20 M_{\odot}$. This result vindicates our assumption that low mass stars (defined here to be those with masses $M_{*}<1 M_{\odot}$ ) have a negligible contribution to the total UV luminosity of the cluster. Similarly, the solid curve in Figure 2 shows the stellar IMF multiplied by the EUV luminosity as a function of mass. This distributed is skewed toward even larger stellar masses, with a peak near $40 M_{\odot}$.

For a given stellar IMF, we thus obtain corresponding distributions of FUV and EUV luminosities. To leading order, these distributions can be characterized by their expectation values and their width (or variance); at a higher order of analysis, however, the shapes of the distribution show significant departures from simple gaussians. For a given IMF and a given UV band (either FUV or EUV), the expectation value $\left\langle L_{U V}\right\rangle_{*}$ of the UV luminosity is determined by the integral

$$
\left\langle L_{U V}\right\rangle_{*}=\int_{1}^{\infty} L_{U V}(m) \frac{d N_{\star}}{d m} d m,
$$

where $d N_{*} / d m=0$ for stellar masses $m>m_{\max }$. The expectation value of equation (4) is normalized so that it provides the expected UV luminosity per star. Because of the wide range of stellar masses and the sensitive dependence of both EUV and FUV emission on stellar mass, this expectation value is much larger than the UV radiation emitted by the majority of stars. As a result, the UV radiation from a cluster will converge to the value implied by this expectation value only in the limit of large $N$ (the value of $N$ necessary to be "large" is determined below). Furthermore, since the EUV radiation depends even more sensitively on stellar mass, this trend is more extreme for the case of EUV radiation (compared to FUV radiation). Small clusters will generally display large departures from the expectation value.

Here we need to determine both the expectation value and variance of the UV luminosity distribution. The UV luminosity is that of the entire cluster, and is given by the sum

$$
L_{U V}(N)=\sum_{j=1}^{N} L_{U V j},
$$


where $L_{U V j}$ is the UV luminosity from the $j t h$ member. In this formulation, we assume that the UV luminosity for a given star is determined solely by the stellar mass, which is drawn independently from a specified stellar IMF. This sum is thus the sum of random variables, where the variables (the individual contributions to the UV power) are drawn from a known distribution, which is in turn determined by the IMF and the $L_{U V}-m$ relation. In the limit of large $N$, the expectation value of the UV power is given by

$$
L_{U V}(N)=N\left\langle L_{U V}\right\rangle_{*}
$$

As usual, the central limit theorem implies that the distribution of values $L_{U V}(N)$ must approach a gaussian form as $N \rightarrow \infty$ (e.g., Richtmyer 1978), although convergence is often slow. One of the issues of interest here is the value of stellar membership $N$ required for these statistical considerations to be valid; similarly, we would like to know the fraction of the cluster population that has such sufficiently large $N$. In its limit of applicability, this gaussian form for the composite distribution is independent of the form of the initial distributions, i.e., it is independent of the stellar IMF and the mass-luminosity relation. The width of the distribution also converges to a known value given by the expression

$$
\langle\sigma\rangle_{U V}{ }^{2}=\frac{1}{N} \sum_{j=1}^{N} \sigma_{j}^{2} \Rightarrow\langle\sigma\rangle_{U V}=\sqrt{N} \sigma_{0}
$$

where $\sigma_{0}$ is the width of the individual distribution, i.e.,

$$
\sigma_{0}^{2} \equiv\left\langle L_{U V}^{2}\right\rangle-\left\langle L_{U V}\right\rangle_{*}^{2}
$$

The expectation values and widths of the luminosity distributions are listed in Table 1 for the four types of stellar IMF used in this paper. The first column gives the parameters $\left(\Gamma, m_{\max }\right)$ of the IMF, where the fraction $\mathcal{F}_{1}=0.12$ for all of the cases. The expectation values are listed as the UV luminosity (either EUV or FUV) per star, and are presented in units of $\mathrm{erg} / \mathrm{s}$ (cgs units). The widths of the distributions are given in normalized form, where $\widetilde{\sigma}_{0 U V} \equiv \sigma_{0 U V} /\left\langle L_{U V}\right\rangle_{*}$. Although the luminosity distributions, as characterized by their width and expectation values, vary somewhat with the stellar IMF, they all show the same general features. For all four IMFs and both UV bands, the expectation values (per star) are $\left\langle L_{U V}\right\rangle_{*} \sim 10^{36} \mathrm{erg} / \mathrm{s}$ in order of magnitude, or about $250 L_{\odot}$. In other words, the mean UV luminosity is always much larger than the total luminosity of the typical star (which has mass $M_{*} \sim 0.5 M_{\odot}$ and luminosity $\sim 0.1 L_{\odot}$ on the main-sequence). This finding is simply a manifestation of the sensitive dependence of the UV luminosity on stellar mass and the large UV luminosities produced by the most massive stars. The second feature illustrated in Table 1 is that the widths of the luminosity distributions are large, roughly $20-40$ times 
wider than the expectation values. This property of the distributions implies that one needs a large number of cluster stars $N$ in order to fully sample the distribution. For example, in order for the width of the distribution to be narrower than the expectation value, the number of stars $N$ must satisfy the relation

$$
N>N_{\min }=\tilde{\sigma}_{0 U V}^{2} \sim 10^{3}
$$

where the numerical value depends on the assumed IMF, but is of order 1000 (see also APFM). Notice that $90 \%$ of the stars in the observed sample in the solar neighborhood are born in clusters with $N<1000$, i.e., the regime of incomplete sampling of the IMF. For the extrapolated distribution of cluster sizes, only about half of the stars are born in the regime of incomplete sampling (see Fig. 1). In either case, a significant fraction of stars are born in clusters where the UV luminosity is subject to incomplete sampling. This finding implies that one must consider the full distribution of possible luminosities - not just mean or median values — when assessing the importance of radiation fields in these environments.

\section{ULTRAVIOLET LUMINOSITY DISTRIBUTIONS}

In this section, we construct the distributions of ultraviolet luminosities. This determination is done for both FUV and EUV radiation, and is carried out in two ways. First, we construct the UV luminosity distribution for clusters of a given size $N$. In this case, each cluster independently samples the stellar IMF $N$ times and the resulting sampling variation leads to a distribution of possible UV luminosities. Second, we construct the UV luminosity distribution for a collection of clusters over a distribution of cluster sizes (namely those shown in Fig. 1). In this latter case, the distribution of UV luminosity (both EUV and FUV) is determined by two input distributions, the stellar IMF and the distribution of cluster membership $f(N)$.

Before constructing the physically relevant luminosity distributions, we first consider the

Table 1: Parameters for UV Luminosity Distributions

\begin{tabular}{lcccc}
\hline \hline IMF $\left(\Gamma, m_{\max }\right)$ & $\left\langle L_{F U V}\right\rangle_{*}(\mathrm{erg} / \mathrm{s})$ & $\tilde{\sigma}_{0 F U V}$ & $\left\langle L_{E U V}\right\rangle_{*}(\mathrm{erg} / \mathrm{s})$ & $\tilde{\sigma}_{0 F U V}$ \\
\hline$(2.35,100)$ & $1.23 \times 10^{36}$ & 25.5 & $8.47 \times 10^{35}$ & 36.9 \\
$(2.1,100)$ & $2.53 \times 10^{36}$ & 18.7 & $1.89 \times 10^{36}$ & 25.5 \\
$(2.5,100)$ & $7.91 \times 10^{35}$ & 30.4 & $5.15 \times 10^{35}$ & 46.2 \\
$(2.35,120)$ & $1.36 \times 10^{36}$ & 26.6 & $1.01 \times 10^{36}$ & 38.5 \\
\hline \hline
\end{tabular}


shapes of the distributions, in particular their substantial departures from gaussianity. To illustrate this behavior, Figure 3 presents the distribution of FUV luminosities, normalized so that the area under the curves equals unity. This plot uses the FUV luminosity divided by the expected mean FUV luminosity for the horizontal axis, and thus emphasizes the shape of the distributions. Keep in mind that the dimensionless widths of the luminosity distributions discussed above (see Table 1) apply to the individual distributions (per star), whereas the distributions in Figure 3 give the shape for different values of $N$. For relatively "small" $N$, i.e., $N=1000$ as shown by the dashed histogram, the distribution is distinctly non-gaussian and peaks at a value significantly less then the mean. For larger $N$, however, the distribution attains the expected gaussian form, as shown by the solid histogram for $N=10^{4}$. The dotted curve shows the corresponding gaussian distribution with the expected dimensionless width for the $N=10^{4}$ case; notice the good agreement. This plot shows that clusters must have relatively large stellar membership, $N$ as large as $N \sim 10^{4}$, before the distribution of FUV luminosity is not dominated by sampling statistics. Note that no clusters this large are observed within $2 \mathrm{kpc}$ (Lada \& Lada 2003), so that the entire solar neighborhood is subject to these sampling variations.

Figure 4 shows the probability distribution for EUV luminosities (Fig. 4a) and FUV luminosities (Fig. 4b) for three different cluster sizes $N$. Note that these distributions (along with those shown in Figures 6 - 9 below) are built using the base 10 logarithm of the luminosity (flux). Specifically, in Figs. 4 and $6-9, P(x) \mathrm{d}\left(\log _{10} x\right)$ (where $x$ is either $L_{U V}$ or $\left.F_{U V}\right)$ is the probability that $x$ falls between $\log _{10} x$ and $\log _{10} x+\mathrm{d}\left(\log _{10} x\right)$. Note that the mean of the logarithm is not the same as the logarithm of the mean, $\left\langle\log _{10} x\right\rangle \neq \log _{10}\langle x\rangle$, so that even a gaussian distribution is skewed (the peak of the distribution falls to the right of mean) when plotted as in Figure 4.

In both Figures $4 \mathrm{a}$ and $4 \mathrm{~b}$, distributions are shown for $N=100$ (dashed curves), $N$ $=300$ (dotted curves), and $N=1000$ (solid curves). As the number of cluster members $N$ increases, the distributions shift to the right, toward higher luminosities, as expected. The distributions also appear to become narrower with increasing $N$. The relative width $\langle\sigma\rangle_{U V} / N\left\langle L_{U V}\right\rangle_{*}$ does indeed become smaller as $N$ grows larger, as outlined above, but the apparent decrease in the total width with $N$ is an artifact of plotting the distribution using a logarithmic scale for the UV luminosity (on the horizontal axis).

Figure 5 shows the UV luminosities for clusters as a function of cluster membership size $N$. Results are shown here for both the EUV luminosities (Fig. 5a) and the FUV luminosities (Fig. 5b). In each case, the luminosity for a given cluster size $N$ shows a wide range of values due to incomplete sampling of the IMF, i.e., the UV luminosity for a given $N$ is characterized by a distribution. The mean value (for a fully sampled IMF) follows 


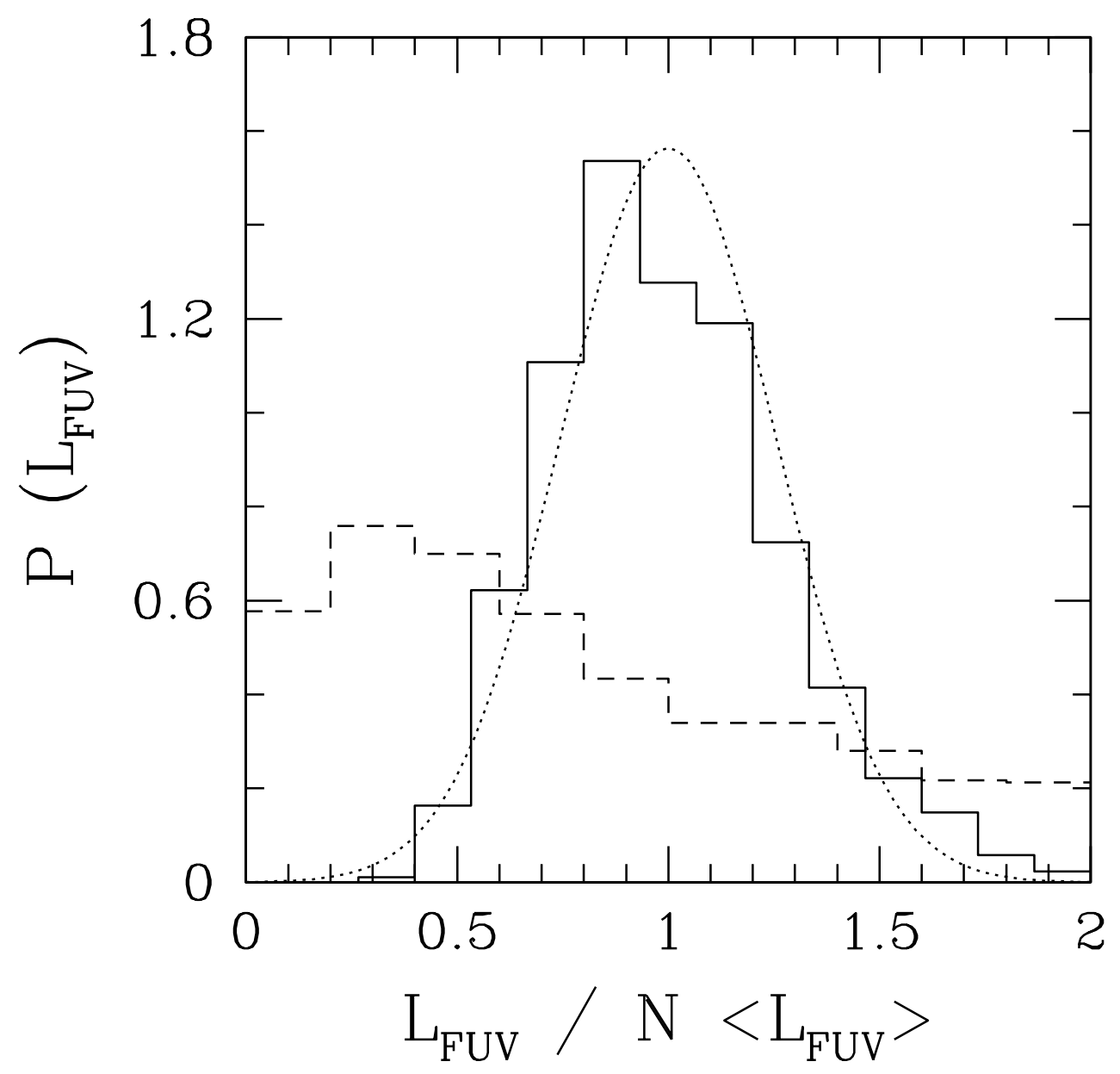

Fig. 3.- Distribution of FUV luminosities. The dashed histogram shows the distribution for clusters with $N=1000$ stars; the solid histogram shows the distribution for $N=10^{4}$. Note that the luminosities (along the horizontal axis) are normalized by the average value one would get with complete sampling of the stellar IMF. The probability distribution (vertical axis) is normalized so that the area under the curves (the total probability) is unity. For comparison, the dotted curve shows a gaussian distribution with width $\sigma=25.5 / \sqrt{10^{4}} \approx$ 0.255 (see Table 1). 


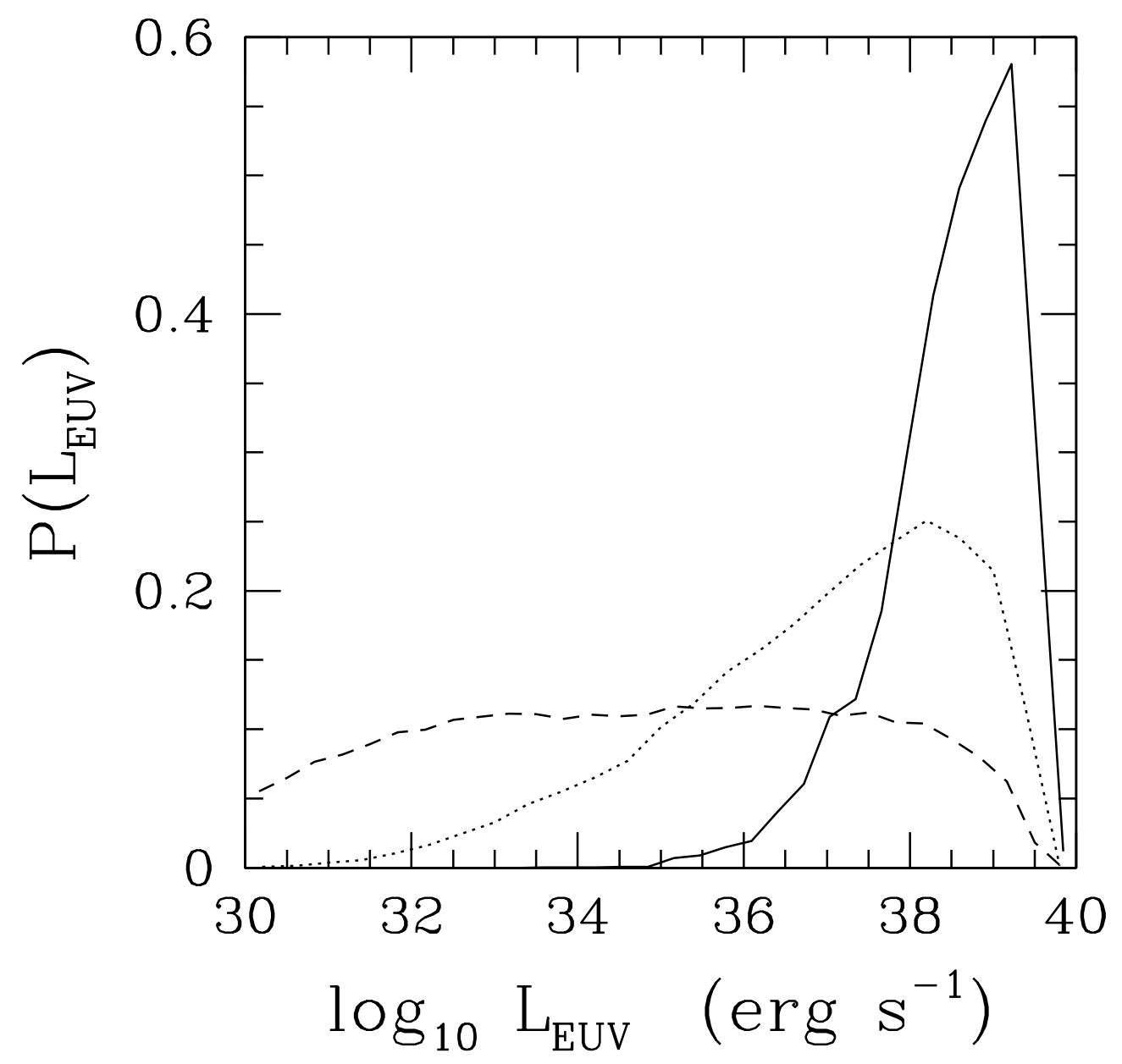

Fig. 4a.- 


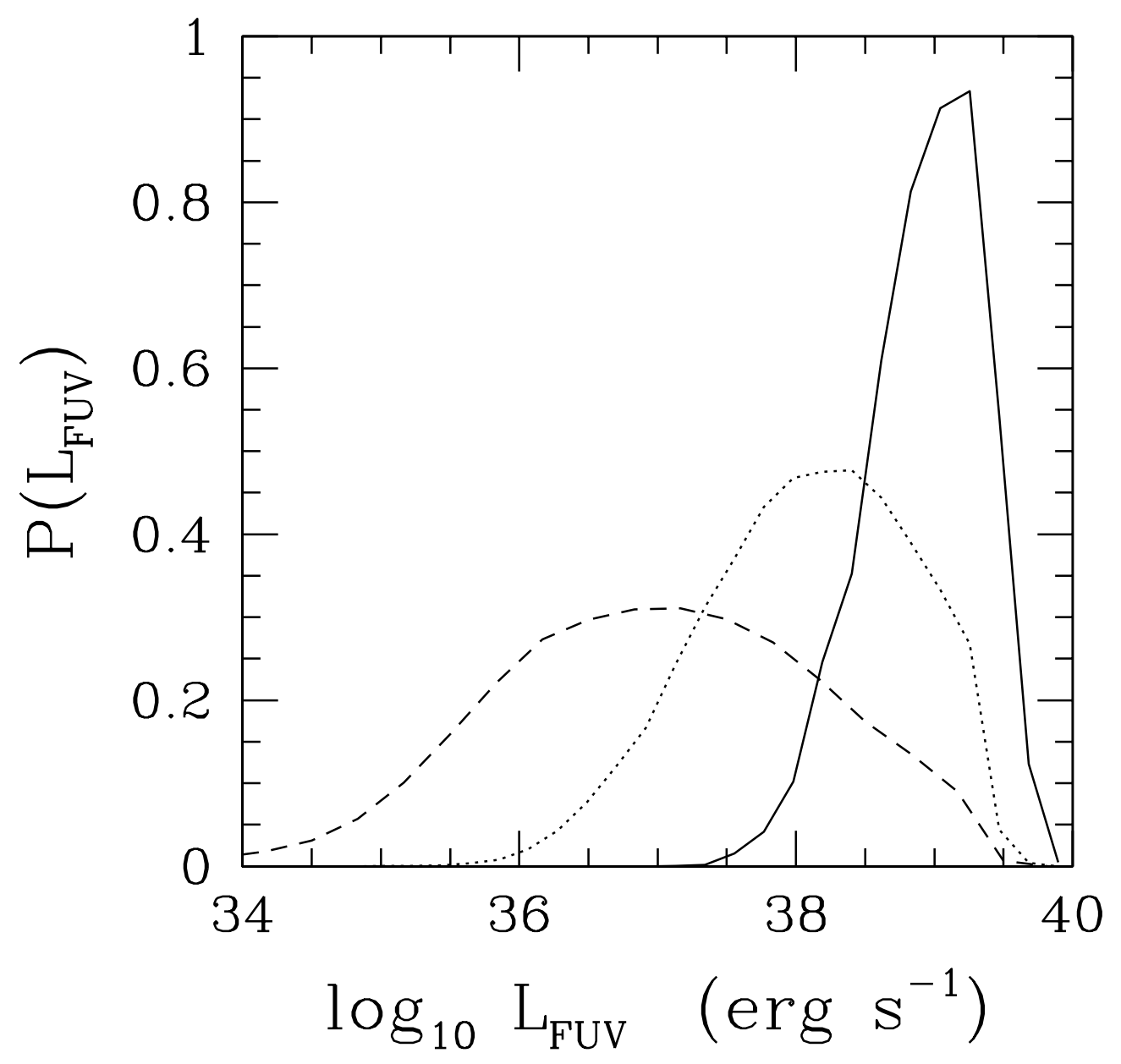

Fig. 4a.- Probability distribution for EUV luminosities. The three curves show the normalized distributions for clusters of varying sizes, with $N=100$ (dashed), $N=300$ (dotted), and $N=1000$ (solid). Each probability distribution is built up from $10^{7}$ independent realizations of the cluster population. b. - Same as Figure 4a, but for FUV luminosities. 
the solid line shown in the Figures. The median value, which is significantly smaller than the mean (for a fully sampled IMF) at small $N$, is shown by the data point symbols. Here the width of the distribution is delineated in two different ways. The error bars show the range of luminosities enclosing the fraction of the distribution from $16.5 \%$ to $83.5 \%$ of the total. The dotted curves show the total expected range of luminosities calculated from the statistical considerations above, i.e., the expectation value plus or minus the width of the distribution for a fully sampled IMF. Since the luminosity distribution is wide, and far from gaussian at low values of $N$, the width of the distribution defined this way is larger than the expectation value. This property of the distributions results in the lower dotted line falling rapidly toward zero (becoming nearly vertical) at $N \approx 1200$ for the EUV distribution and at $N \approx 700$ for the FUV distribution. These results are in basic agreement with those obtained earlier for EUV radiation (Armitage 2000) and FUV radiation (APFM).

The results depicted in Figure 5 show that the distributions of UV luminosity have qualitatively different behavior for large $N$ and small $N$ clusters. Note that the ranges of expected luminosity values become centered on the expectation values for sufficiently large values of stellar membership $N$. For the EUV distribution, this centering occurs for $N \gtrsim 2000$, whereas for the FUV distribution it occurs for $N \gtrsim 1000$. For both cases, this centering occurs at somewhat larger $N$ than the values required for the distributions to be narrower than their expectations values. By coincidence, the required "centering" values are roughly the same as the value of $N$ for the largest cluster (the Orion Nebula Cluster, or ONC) in the solar neighborhood cluster distribution. In other words, for all of the clusters in the solar neighborhood, the stellar membership is not large enough to use statistical arguments to predict expectation values, etc., so that the central limit theorem does not fully apply (i.e., the limit of large $N$ is not reached). The distributions thus depend sensitively on the sampling of the underlying IMF. Notice that this finding makes sense: For $N \sim 1000$, say, the number $N_{U V}$ of stars large enough to provide any significant UV luminosity is only about $N_{1} \sim \mathcal{F}_{1} N \sim 120$, whereas the number $N_{20}$ of stars larger than $20 M_{\odot}$ (where most of the UV is emitted - see Fig. 2) is only $N_{20} \sim N \mathcal{F}_{1}(20)^{-1.35} \sim 2$. In other words, in rough terms, only clusters with stellar membership $N$ greater than $\sim 1000$ are large enough to populate the part of the IMF where most of the UV radiation is emitted.

The distributions presented thus far have been constructed using the observed distributions of cluster sizes $N$ in the solar neighborhood (Lada \& Lada 2003). We now consider the effects of variations in the cluster size distribution on the luminosity distributions. Specifically, we first sample the cluster size distribution to determine the size $N$ of the cluster to which a "test" star belongs, and then sample the IMF N times to determine the corresponding luminosity of our "test" star's cluster. We repeat this process a total of $10^{7}$ times to then build up luminosity distributions (we have varied the sampling size and verified that it 


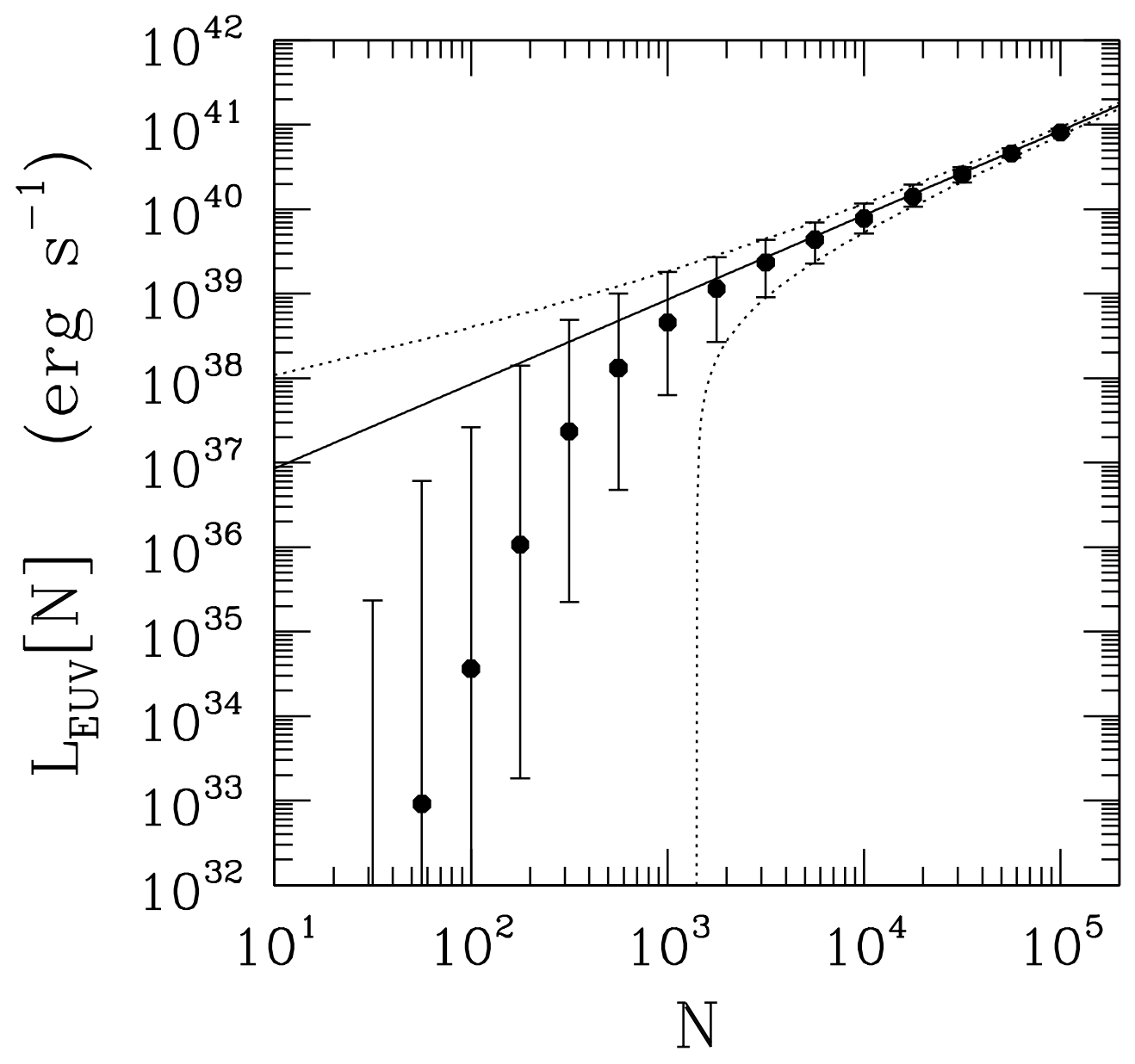

Fig. 5a.- 


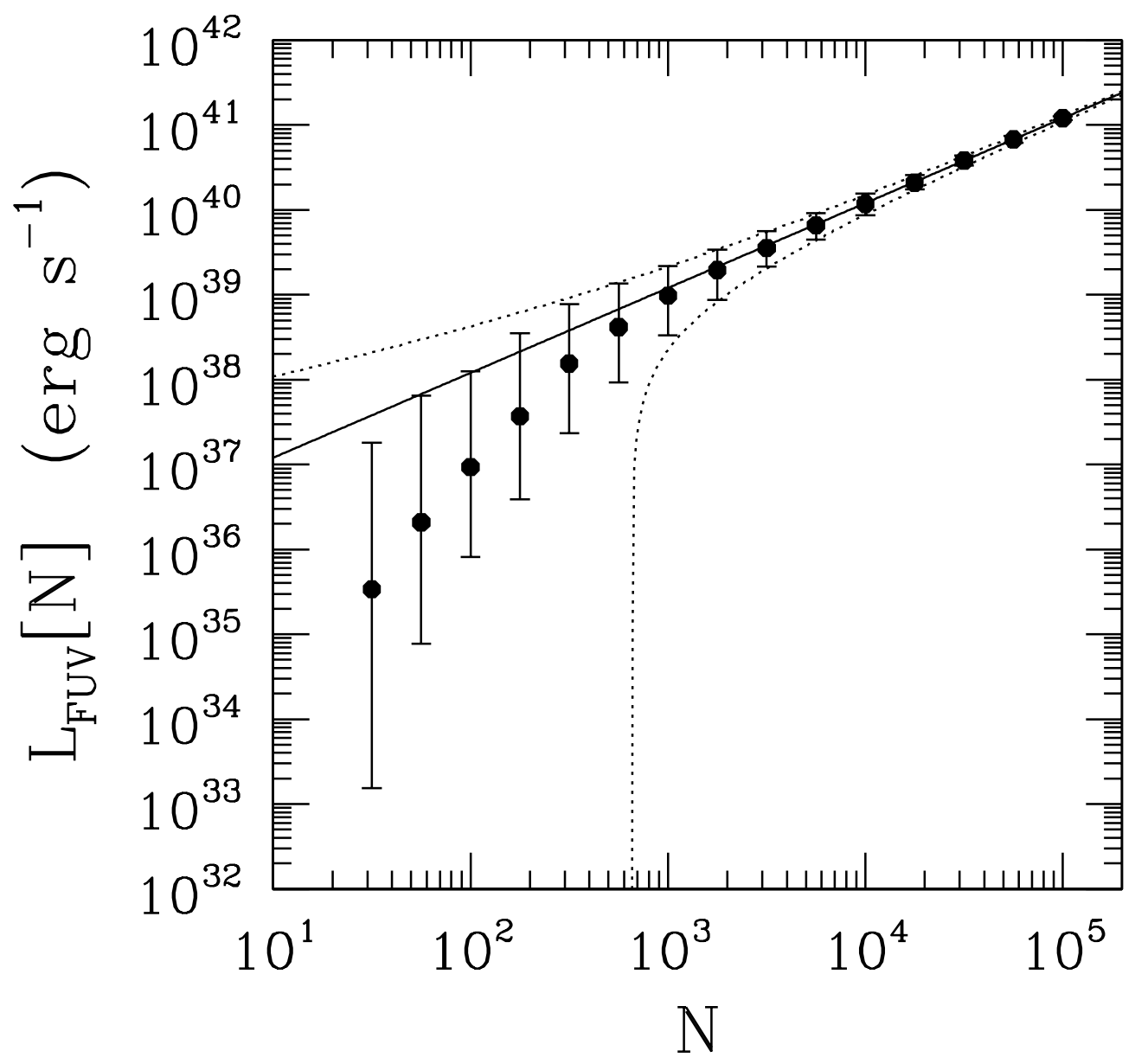

Fig. 5a.- EUV Luminosity per cluster as a function of cluster size $N$. For each value of $N$, the EUV luminosity can have a wide distribution of values. The solid line shows the mean value for a fully sampled IMF $\left(N\left\langle L_{U V}\right\rangle_{*}\right)$ as a function of cluster membership $N$. The data points show the median values of the distribution, where the error bars delineate the range from 0.165 to 0.835 . The dotted curves show the expectation value plus or minus the width of the distribution for a fully sampled IMF. b. - Same as Figure 5a, but for the FUV Luminosity per cluster. 
is large enough for convergence). Figure 6 shows a comparison of the resulting luminosity distributions for different cluster size distributions, including the solar neighborhood (solid curve) and the extended distribution that is extrapolated up to cluster sizes of $N=10^{5}$ (dashed curves). The EUV distributions are shown in Figure 6a, and the FUV distributions are shown in Figure 6b. A related issue is the extent to which the UV radiation fields are dominated by the most massive star in the cluster. To consider this problem, both panels of Figure 6 also show the distributions of luminosity using only the most massive star in the cluster (dotted curves), for both the solar neighborhood and extended distributions of cluster sizes. For the solar neighborhood distribution, which includes only relatively "small" clusters, the distribution of luminosity with only the most massive star and the distribution with all stars are nearly the same; this result indicates that the UV radiation fields are dominated by the single most massive star in such systems. In the case of the extended cluster size distribution, however, there is marked difference between the distribution that includes all stars and the one that includes only the most massive star. This trend is clearly evident for both the EUV and FUV bands, and shows that many stars provide a significant contribution to the UV luminosity for large clusters.

\section{ULTRAVIOLET FLUX DISTRIBUTIONS}

Given the distribution of UV luminosity, both for clusters of a given size $N$ and for the expected distribution of clusters, the next step is to construct distributions of UV flux. To convert the distributions of EUV and FUV luminosities into fluxes, we first need to specify the distribution of stars within clusters. In other words, we must convolve the distribution of luminosities with a distribution of radial positions to produce a distribution of fluxes. For most of this paper, we assume that the stars follow a distribution of radial positions that corresponds to a density law of the form $\rho_{*} \propto 1 / r$, or, equivalently, $d m \propto r^{2} \rho_{*} d r \propto r d r$. Although the density profile will have an inner cutoff (to remove the apparent singularity at the origin), this scale does not enter into the problem (one can integrate over it). However, the outer boundary does play a role and here we truncate the distribution at the outer radius $R_{\mathrm{c}}$ of the cluster, where $R_{\mathrm{c}}$ is given as a function of $N$ in equation (11). This form for the density distribution of stars is consistent with results from N-body simulations of analogous clusters (see eq. [3] and Table 2 of APFM). Note that by considering the distribution of radial positions to determine flux, we are determining the complete ensemble of possible flux values provided by the cluster environment. However, individual solar systems will execute orbits within the cluster potential, and the distribution of orbit-averaged fluxes will be somewhat narrower. We take up this issue in $§ 4.4$. 


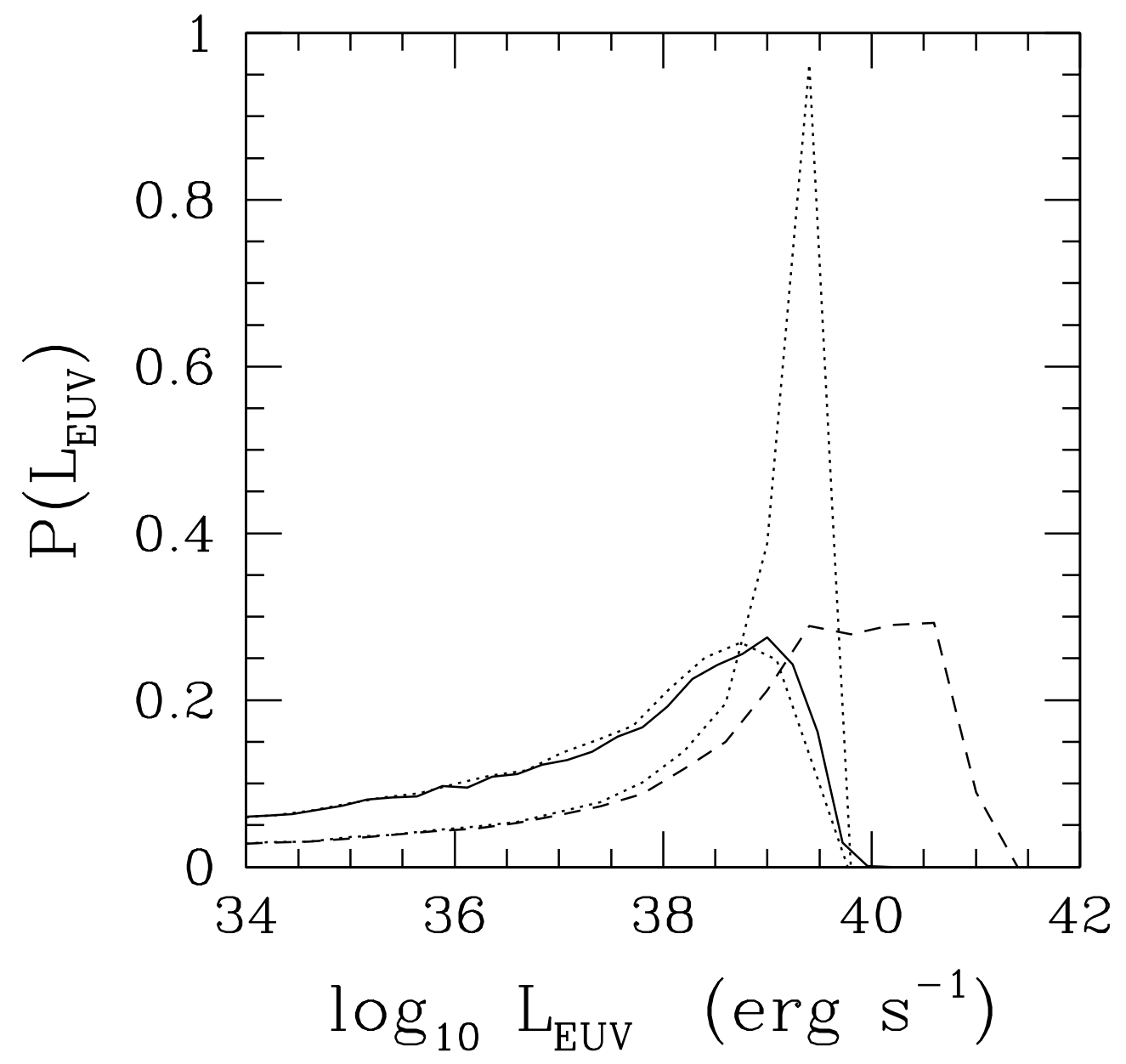

Fig. 6a.- 


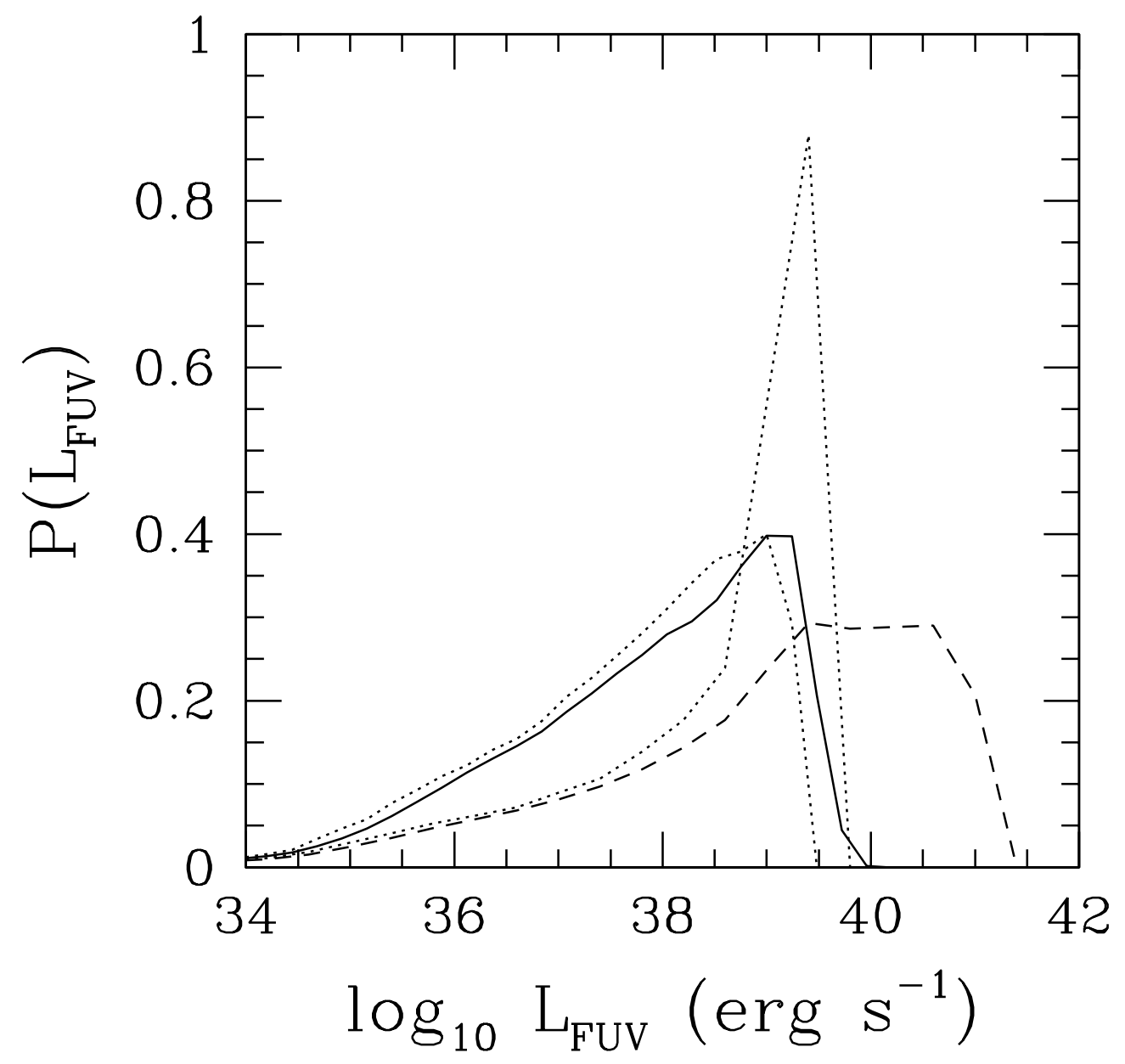

Fig. 6a.- Distributions of EUV luminosities for different cluster samples. The solid line shows the distribution of EUV luminosity for the sample of clusters observed in the solar neighborhood (Lada \& Lada 2003). The dashed curve shows the corresponding distribution of EUV luminosities for the extended cluster distribution that extends up to $N=10^{5}$. The dotted curves show the analogous distributions for the case in which only the most massive star is allowed to contribute to the EUV luminosity of the cluster. b. - Same as Figure 6a, but for FUV luminosities. 


\subsection{Extinction}

In this treatment, we provide flux distributions both with and without extinction. Since the gas (and hence the dust) in young clusters has a relatively short lifetime, perhaps only $\sim 3 \mathrm{Myr}$ (Allen et al. 2007), the flux distributions with no extinction will be applicable for much of the time. Nonetheless, we want to consider how much extinction can change the radiation fields. In order to consider the effects of extinction on the expected radiation fields, we need to specify the distribution of gas (and dust), as well as the opacity at UV wavelengths. For the sake of definiteness, we take the gas distribution to follow a Hernquist profile (Hernquist 1990) so that the density is given by

$$
\rho=\frac{\rho_{0}}{\xi(1+\xi)^{3}}
$$

where $\xi=r / r_{s}$ and $r_{s}$ is the scale radius of the profile. Here we take $r_{s}=R_{\mathrm{c}}$ so that the density distribution has the approximate form $\rho \propto 1 / r$ for radii within the cluster itself. The form of equation (10) allows the density and its corresponding gravitational potential to match smoothly onto the background of the molecular cloud, but otherwise plays no role. The density scale $\rho_{0}$ is determined by the specification of the gas content of the cluster. For example, if the star formation efficiency $\epsilon=1 / 3$, so that two thirds of the mass within

$R_{\mathrm{c}}=r_{s}$ is made of gas, then $\rho_{0}=4 N\left\langle M_{*}\right\rangle / \pi r_{s}^{3}$, where $\left\langle M_{*}\right\rangle$ is the mean stellar mass of the population.

With the density specified by equation (10), the column density is given by the integral

$$
N_{\mathrm{col}}(r)=\frac{1}{\langle\mu\rangle} \int_{r_{1}}^{r} \rho(r) d r=\frac{\rho_{0} r_{s}}{\langle\mu\rangle} \int_{\xi_{1}}^{\xi} \frac{d \xi}{\xi(1+\xi)^{3}},
$$

where $\langle\mu\rangle$ is the mean mass of the particles and where $r_{1}$ is an inner cutoff radius. In most clusters, as assumed herein, the most massive star lies at the cluster center and will evacuate its immediate vicinity and produce an inner cutoff radius. Here we set the inner cutoff radius by assuming that the evacuated cavity originally contained a mass $Q M_{* \max }$, leading to an inner radius

$$
r_{1}=\left(\frac{Q M_{* \max }}{2 \pi \rho r_{s}^{3}}\right)^{1 / 2}
$$

where we use $Q=3$ and 10 to define two choices of $r_{1}$.

The integral from equation (11) can be evaluated to obtain the result

$$
N_{\mathrm{col}}=\frac{\rho_{0} r_{s}}{\langle\mu\rangle}\left\{\ln \left[\frac{\xi\left(1+\xi_{1}\right.}{\xi_{1}(1+\xi)}\right]+\frac{1}{2(1+\xi)^{2}}-\frac{1}{2\left(1+\xi_{1}\right)^{2}}+\frac{1}{1+\xi}-\frac{1}{1+\xi_{1}}\right\},
$$

where $\xi=r / r_{s}$ and $\xi_{1}=r_{1} / r_{s}$. 
Next we want to obtain a general assessment of the effects of extinction on the distribution of the UV radiation fields. We assume that all of the UV-generating stars lie within the spherical cavity defined above. The target systems are distributed according to a $\rho_{*} \sim 1 / r$ distribution within the radial range $0 \leq r \leq R_{c}(N)$, i.e., the target stars can orbit through the evacuated central cavity and experience no extinction (even though they do not form there). Each radial position has both an associated flux and an associated column density $N(r)$ as defined by equation (13). The column density can be converted into an optical depth through the relation

$$
\tau_{U V}=\sigma_{U V} N_{\mathrm{col}}
$$

where the cross sections are given by $\sigma_{F U V}=10^{-21} \mathrm{~cm}^{2}$ and $\sigma_{E U V}=2 \times 10^{-21} \mathrm{~cm}^{2}$.

Note that actual embedded clusters will not necessarily have smooth distributions of column density and hence extinction. The dynamic nature of the star formation process (e.g., winds, jets, and outflows), and the cloud formation process itself, lead to a highly clumpy geometry with some less obscured lines of sight. In such a system, the degree of penetration of UV radiation, and the corresponding photo-ionization rates, are much larger than in the case of uniform density distributions (Bethell et al. 2007). More detailed radiative transfer models of this type should be performed for these cluster environments. One should also keep in mind that the gas only resides in the cluster for a relatively short time (about 3-4 Myr; Allen et al. 2007), so the full (unattenuated) UV flux distribution will be applicable for much of the time. The treatment presented here thus represents an upper limit to the effects of extinction on the distribution of UV fluxes. Nonetheless, as shown next, this effect can be significant.

\subsection{Benchmarks}

One important issue is to determine how often the radiation field impinging on circumstellar disks is dominated by the background cluster or by the central star itself. For any given star and given background radiation flux, we can determine the radius within the disk where the UV radiative flux from the central star is equal to that of the background. For a given central star, let $L_{U V *}$ be the stellar luminosity within a UV band, either FUV or EUV, and let $F_{U V}$ be the flux in the same UV band from the background environment of the cluster. At a given radius $\varpi$ within the disk, the UV flux contribution from the central star is equal to that of the background $\left(F_{U V}\right)$ when

$$
\frac{L_{U V *}}{4 \pi \varpi^{2}}=F_{U V} .
$$




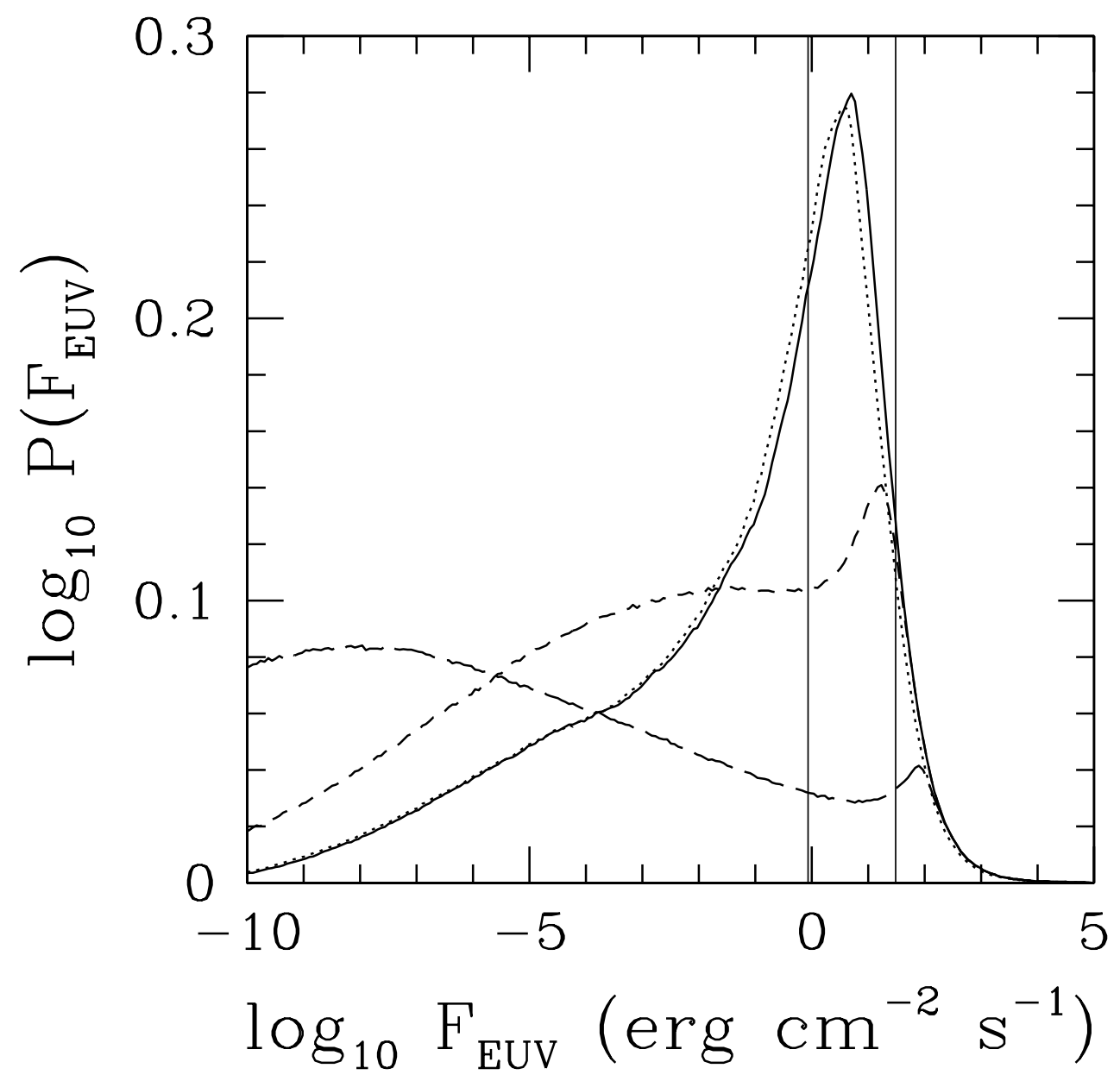

Fig. 7a.- 


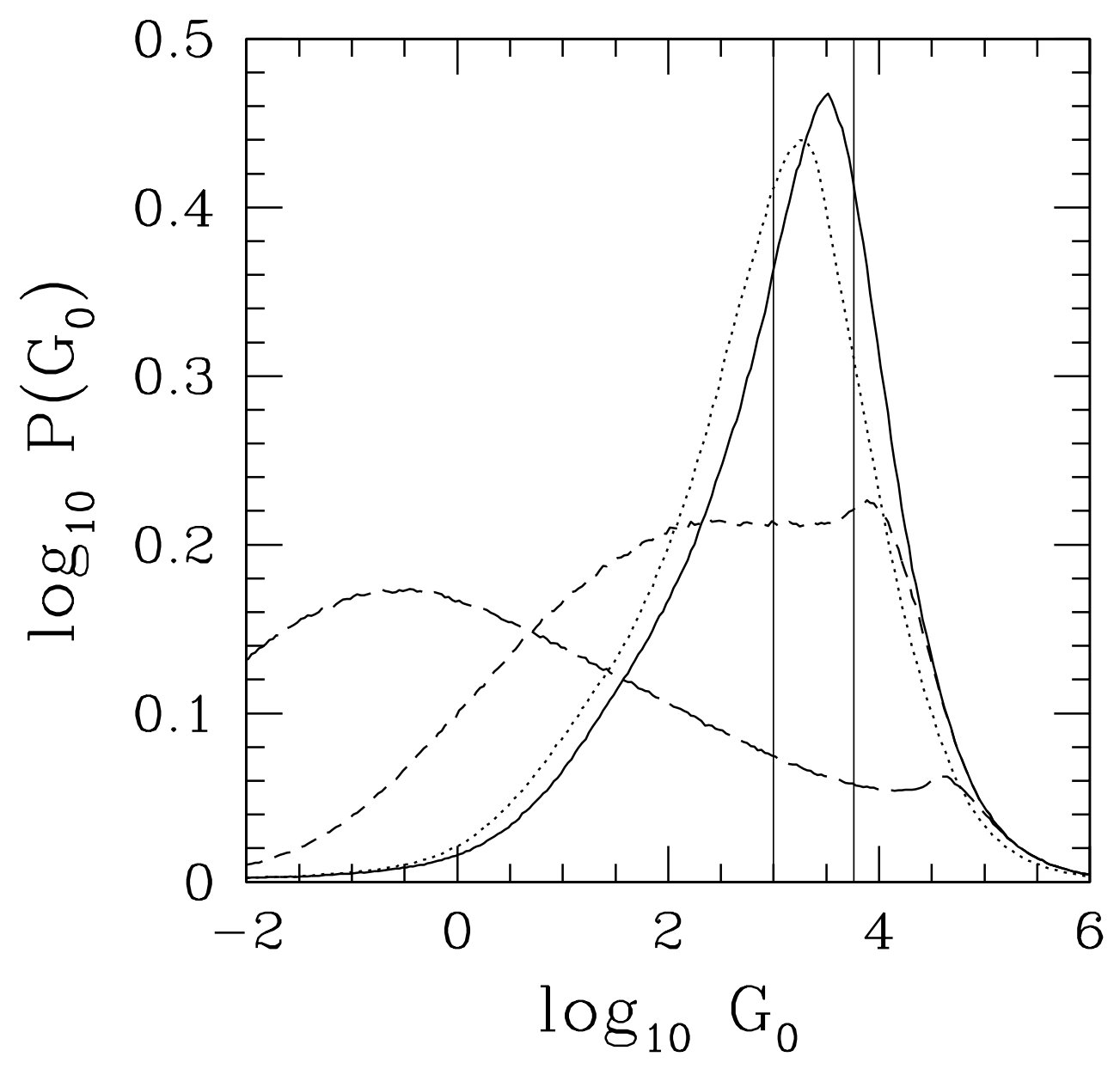

Fig. 7a.- Distribution of EUV flux as a function of EUV flux $F_{E U V}$ determined by sampling over the Lada \& Lada (2003) distribution of cluster sizes. This case uses the first stellar $\operatorname{IMF}$ with $\left(\mathcal{F}_{1}, \Gamma, m_{\max }\right)=(0.12,2.35,100)$. The solid curve shows the full distribution; the dotted curve shows the reduced distribution using only the radiation from the most massive star. The dashed curves show the flux distribution with extinction included for $Q$ $=3$ (long-dashes) and $Q=10$ (short-dashes). The vertical lines mark the benchmark flux values for which the background cluster radiation dominates that of the central star (left) and for which the disk is evaporated in $10 \mathrm{Myr}$ (right). b. - Same as Figure 7a, but for FUV flux. 
The central star dominates at smaller radii and the background cluster dominates at larger radii. In order to provide a benchmark for comparison, we must specify the radius $\varpi$ of interest. Within our own solar system, planet formation takes place within $30 \mathrm{AU}$; in more general solar systems, the time scale for forming planets increases with radius and the lifetime of the gas decreases with radius, so we expect planet formation to become increasingly difficult for larger values of $\varpi$. We thus adopt $\varpi=30$ AU to evaluate equation (15). The resulting benchmark flux is thus approximately $F_{E U V}$ (bench) $\approx 0.86 \mathrm{erg} \mathrm{cm}^{-2} \mathrm{~s}^{-1}$ for the EUV band and $G_{0}$ (bench) $\approx 1000$ for the FUV band. Note that throughout this paper, we present FUV fluxes in units of $G_{0}$, where $G_{0}=1$ corresponds to the "standard" interstellar value of $1.6 \times 10^{-3} \mathrm{erg} \mathrm{s}^{-1} \mathrm{~cm}^{-2}$.

One of the most important effects of the background radiation fields is to drive photoevaporation from circumstellar disks, thereby leading to loss of planet-forming potential. We thus want to determine what part of the expected distribution of UV flux will lead to substantial mass loss. For the sake of definiteness, we find the flux required to evaporate a $M_{d}=0.05 M_{\odot}$ disk over at time scale of $10 \mathrm{Myr}$. The expected mass loss rate $\dot{M}$ from a circumstellar disk exposed to EUV radiation can be written in the form

$$
\dot{M} \approx 10^{-8} M_{\odot \mathrm{yr}^{-1}}\left(\frac{F_{E U V}}{130 \operatorname{erg~cm} \mathrm{cm}^{-1}}\right)^{1 / 2}\left(\frac{r_{d}}{30 \mathrm{AU}}\right)^{3 / 2}
$$

where $r_{d}$ is the disk radius (Hollenbach et al. 2000). Note that a mass loss rate of $10^{-8} M_{\odot}$ $\mathrm{yr}^{-1}$ will evaporate a typical planet-forming disk with mass $M_{d}=0.05 M_{\odot}$ in only about 5 Myr. As a result, EUV fluxes of order $F_{E U V} \sim 30 \mathrm{erg} \mathrm{cm}^{-2} \mathrm{~s}^{-1}$ can evaporate this type of disk in $10 \mathrm{Myr}$ and can thereby compromise the planet formation process.

For FUV radiation, the detailed models indicate that an external radiation field of $G_{0}$ $=3000$ will evaporate a disk around a $1.0 M_{\odot}$ star down to $36 \mathrm{AU}$ over a time of $10 \mathrm{Myr}$, where the assumed disk mass $M_{d}=0.05 M_{\odot}$ (Adams et al. 2004). Interpolating between the published models for $G_{0}=3000$ and those for $G_{0}=30,000$, we estimate that a radiation flux of $G_{0}=5800$ will evaporate the disk down to $30 \mathrm{AU}$ in $10 \mathrm{Myr}$. Note that this benchmark flux is only about 6 times larger than the flux required to dominate the stellar FUV flux (see above), i.e., a relatively modest increase in the FUV radiation environment can lead to a significant effect on forming solar systems. As noted in $\S 2$, the use of zero-age main sequence luminosity values is not entirely appropriate in this context given that the most massive stars (those with $m \sim 100$ ) burn their hydrogen in less than 10 million years. However, stellar evolution results in two competing effects for the total cluster luminosity, since stars get brighter as they age, but the most massive stars have relatively short lifetimes. Our results are therefore expected to be representative of the actual values. 


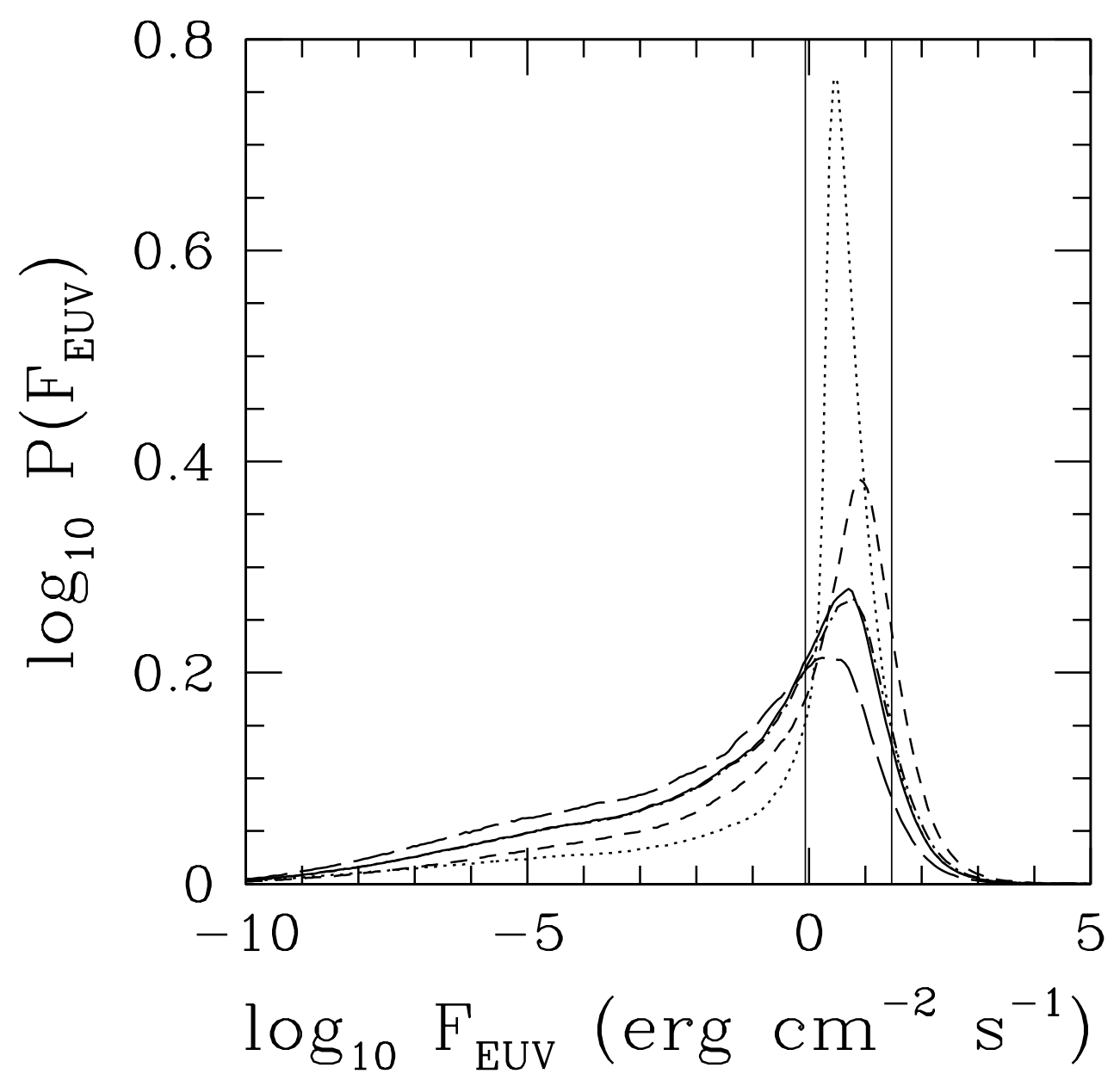

Fig. 8a.- 


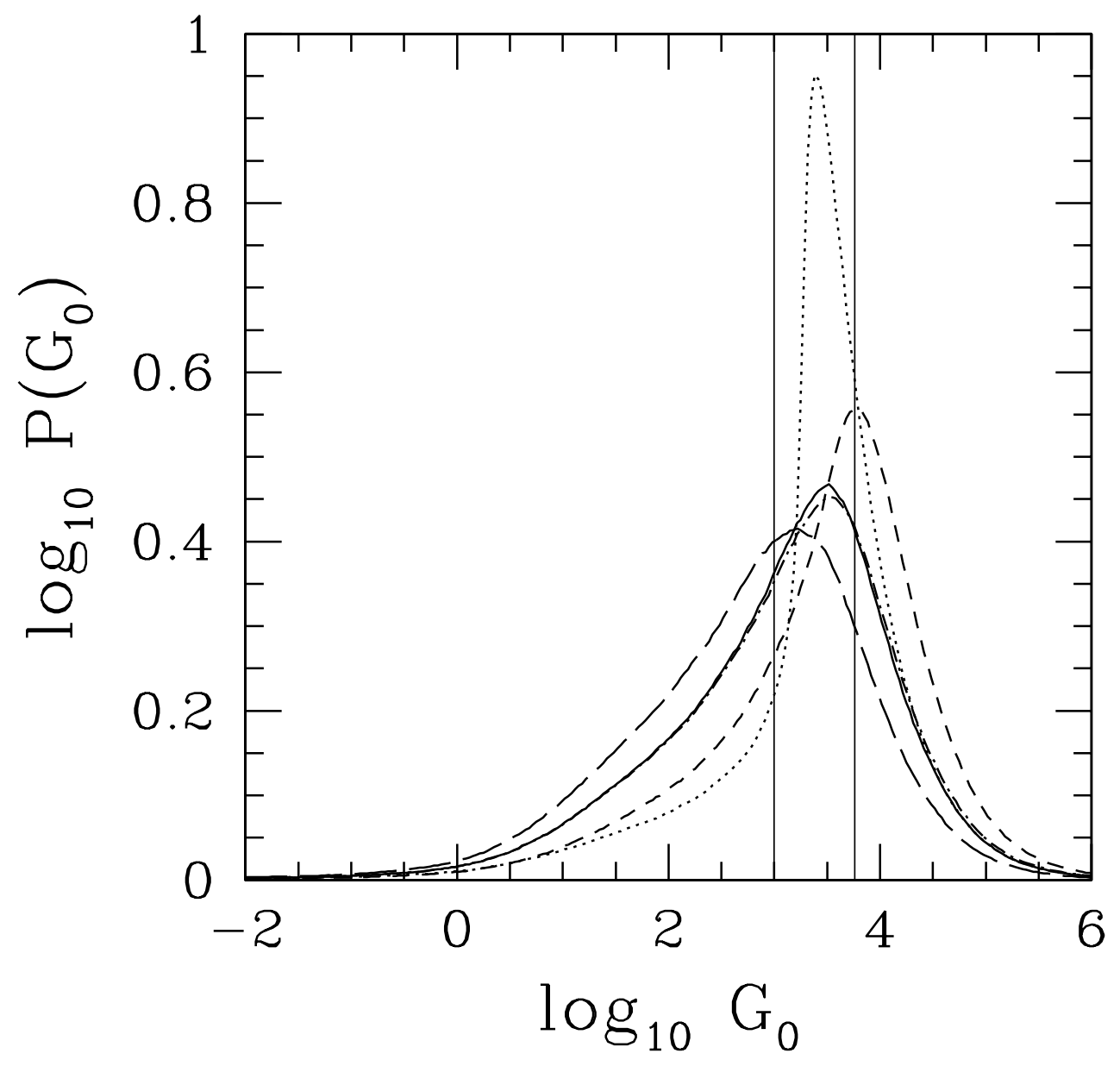

Fig. 8a.- EUV flux distributions for different stellar IMFs and cluster size distributions. For the standard cluster size distribution in the solar neighborhood, the resulting flux distributions are shown for stellar IMFs with parameters $\left(\Gamma, m_{\max }\right)$ given by $(2.35,100)$ (solid curve) $(2.1,100)$ (short dashed curve), $(2.5,100)$ (long dashed curve), and $(2.35,120)$ (dotdashed curve). The dotted curve shows the flux distribution for the standard IMF (2.35, $100)$ and the extended cluster size distribution. The vertical lines mark the benchmark flux values for which the background cluster radiation dominates that of the central star (left) and for which the disk is evaporated in $10 \mathrm{Myr}$ (right). b. - Same as Figure 8a, but for FUV flux. 


\subsection{Composite Flux Distributions}

Figure 7 shows the flux distributions for the expected cluster sample. Note that these distributions are determined by first sampling the cluster size distribution (for the solar neighborhood), then sampling the standard stellar IMF $N$ times for a cluster of given size $N$, and finally sampling the radial positions according to a $\rho \sim 1 / r$ distribution. This process is repeated to build up the distributions shown herein. The EUV distributions are shown in Figure 7a, and the FUV distributions are shown in Figure 7b. For both of these cases, the solid curve shows the full distribution for the case of no extinction; the dashed curve shows the distribution with extinction included. Also shown in the Figures are the corresponding distributions for the radiation produced by the most massive star in the cluster alone (with no extinction). These latter distributions are almost identical to the full distributions; this finding indicates that the radiation fields are dominated by the most massive star in the cluster for the ensemble of clusters represented in the solar neighborhood. The dashed curves in Figure 7 show the effects of including extinction, for the two cases $Q=3$ (long dashes) and $Q=10$ (short dashes).

The vertical lines in Figure 7 depict the benchmark flux values defined in the previous section, i.e., the values for which the background cluster radiation dominates that of the central star (at disk radius $30 \mathrm{AU}$ ) and the values for which the disk is evaporated in 10 Myr (for a disk with mass $M_{d}=0.05 M_{\odot}$ and radius $r_{d}=30 \mathrm{AU}$ ). The former benchmark fluxes are smaller than the latter for both the EUV and FUV distributions. For the case of EUV radiation, 42 percent of the distribution has the radiation field dominated by the background cluster, but only 7 percent of the distribution is exposed to enough EUV radiation to evaporate the disks. For the case of FUV radiation, effect is somewhat larger, with 58 percent of the distribution being dominated by the background cluster and 25 percent of the distribution exposed to enough FUV radiation to drive substantial disk evaporation. These results thus show that disk evaporation tends to be dominated by FUV radiation, rather than EUV radiation, in agreement with previous claims (Hollenbach et al. 2000; Adams et

al. 2004). Furthermore, the dominant source of (EUV) ionization in circumstellar disks (as required for the magneto-rotational instability, MRI, for example) is usually the central star rather than the background (note that cosmic rays and X-rays also provide ionization and thereby affect MRI).

Figure 8 shows the effects of varying the stellar IMF and the assumed cluster size distribution on the resulting composite UV flux distributions. Results are shown for both EUV radiation (Fig. 8a) and FUV radiation (Fig. 8b). No extinction has been included in the construction of these distributions. For each case, four of the curves show the distributions for the four different stellar IMFs used in this paper. The upper mass cutoff has relatively 
little effect on the composite flux distribution. This finding indicates that the stellar IMF is sufficiently steep so that stars with the highest masses do not dominate the UV radiation output. This result is consistent with the distributions shown in Figure 2, which indicate that the UV contribution peaks at masses of $20-40 M_{\odot}$. The slope of the IMF has a larger effect on the flux distributions, with the expected result: A shallow slope leads to more high mass stars and shifts the UV flux distributions to higher values (to the right), whereas a steeper slope works in the opposite direction.

The fifth (dotted) curves in Figure 8 shows the resulting composite flux distribution for the extended cluster size distribution (and the standard stellar IMF). The flux distributions are significantly narrower for the extended cluster size distribution. Notice that the peak of the distribution does not change substantially. The reason for this invariance can be understood as follows: First, note that the cluster size distribution used here corresponds to equal numbers of stars being found in each decade of cluster size; as a result, the extended cluster distribution has only about half of its stars in the large $N$ clusters, i.e., the clusters that are added to the distribution for the solar neighborhood. For these (additional) large $N$ clusters, the IMF sampling is relatively complete so that the total luminosity of a cluster obeys the scaling $L_{U V}($ tot $) \sim N\left\langle L_{U V}\right\rangle_{*}$; however, the cluster radius scales according to equation (11), and most stars in the cluster reside at the larger radii, so that $r^{2} \sim N$, and hence the flux $F_{U V} \sim L_{U V}(t o t) / 4 \pi r^{2}$ becomes nearly independent of $N$.

\subsection{Orbits}

The distributions of flux considered above were constructed by statistically sampling the distribution of radial positions within a cluster. However, any given solar system will follow particular orbits through the cluster. In this subsection, we consider the interplay between orbital motion and radiation exposure of circumstellar disks.

The first task is to find the orbit-averaged radiative flux. For the density profile of equation (10), the potential is given by

$$
\Psi=\frac{\Psi_{0}}{1+\xi}
$$

where $\xi=r / r_{s}$ as above and where $\Psi_{0}=2 \pi G \rho_{0} r_{s}^{2}$ determines the total depth of the potential well. If we define $M_{T}$ to be the total mass enclosed within the scale radius $r_{s}$, which is taken here to be the cluster radius $R_{c}(N)$, then $\Psi_{0}=4 G M_{T} / r_{s}$. Following previous treatments (Adams \& Bloch 2005, APFM), we define dimensionless energy and angular momentum 
variables

$$
\epsilon \equiv \frac{|E|}{\Psi_{0}} \quad \text { and } \quad q \equiv \frac{J^{2}}{2 \Psi_{0} r_{s}^{2}},
$$

where $E$ and $J$ are the (physical) specific energy and specific angular momentum of the orbit. As shown in APFM, the radiation flux averaged over an orbit is then given by the expression

$$
\left\langle F_{U V}\right\rangle_{\text {orb }}=\frac{L_{U V}}{8 r_{s}^{2}} \frac{A(q) \epsilon^{3 / 2}}{\cos ^{-1} \sqrt{\epsilon}+\sqrt{\epsilon} \sqrt{1-\epsilon}},
$$

where $A(q)$ is a slowly varying function of angular momentum and is constrained to lie in the range $1 \leq A(q) \leq \sqrt{2}$.

Equation (19) gives the orbit-averaged flux for a orbit with given energy $\epsilon$ and angular momentum $q$. The stellar dynamics of the cluster determines the distribution of energy and angular momentum for the cluster members (e.g., Binney \& Tremaine 1987; hereafter BT87). In particular, for given assumptions about the velocity distribution, one can find the relationship between the distribution function, the differential energy distribution, and the density profile of the cluster. We assume an isotropic velocity distribution and a density profile form $\rho \propto 1 / r$; note that this density profile is consistent with our N-body simulations of young embedded clusters (APFM). For this case, the differential energy distribution the probability distribution for orbital energies — takes the form

$$
h(\epsilon)=\frac{d P_{m}}{d \epsilon}=\frac{2}{\left(1-\epsilon_{0}\right)^{2}}(1-\epsilon),
$$

which is normalized for the range of dimensionless energies $\epsilon_{0} \leq \epsilon \leq 1$. In these systems, stellar orbits are not highly populated for low energies, those well beyond the starting cluster radius, so we truncate the distribution at some energy scale $\epsilon_{0}$. For example, the energy corresponding to a radial orbit with its outer turning point at twice the nominal cluster radius has $\epsilon=1 / 3$, which thus defines a representative value. Here we use $\epsilon_{0}=1 / 2,1 / 3$, and $1 / 4$ to sample the possible values.

The distribution of energies within the cluster (eq. 20]), in conjunction with the orbitaveraged flux for a given energy (eq. [19]), define a distribution of fluxes within a given cluster (with given UV luminosity $L_{U V}$, which can be taken to be either EUV or FUV). Note that the dependence of the orbit-averaged flux on angular momentum $(q)$ is weak, and that the variations tend to average out over the ensemble of possible values. We thus need to adopt an intermediate value for $A(q)$ in equation (19). The convolution of equations (19) and (20) shows that the resulting distribution of orbit-averaged flux is weighted toward the lowest flux values, those corresponding to the lowest energies $\epsilon$, or, equivalently, the outer parts of the cluster. This result makes sense because the mass profile $M(r) \sim r^{2}$, so most of the stars must spend most of their time in the outer realm of the cluster system. 


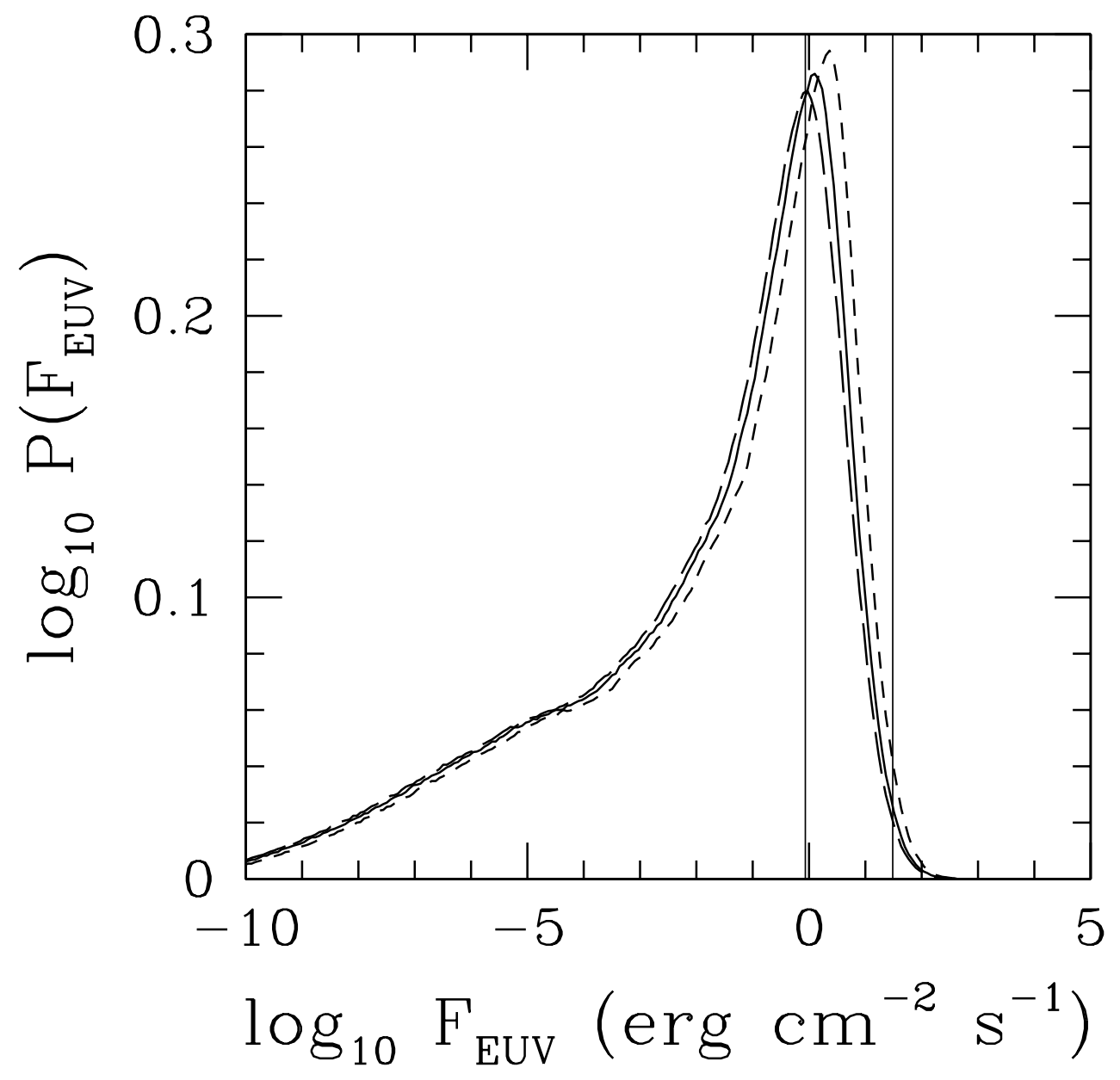

Fig. 9a.- 


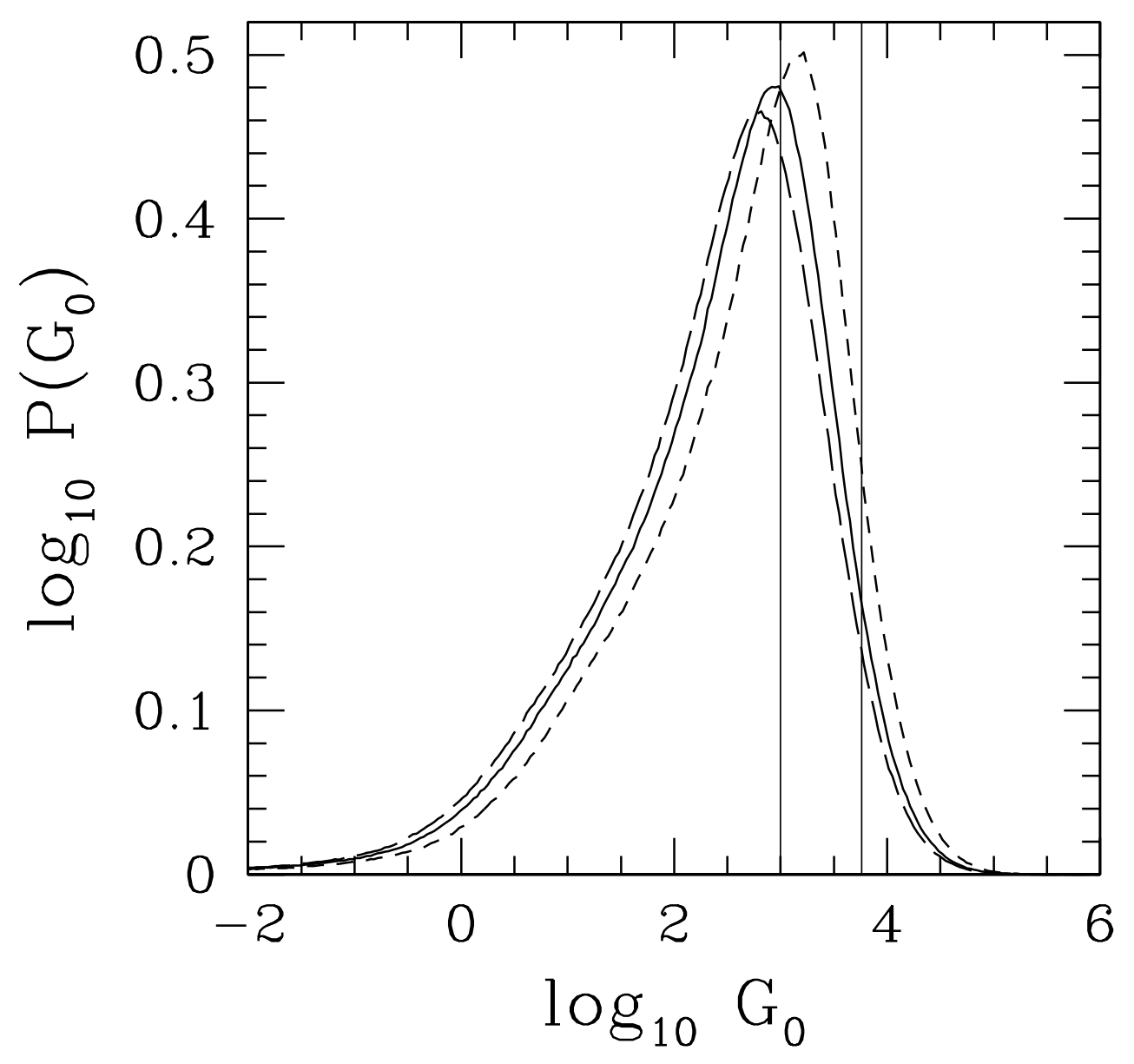

Fig. 9a.- Distribution of orbit-averaged EUV flux. (Compare with Fig. 7a). This case uses the standard stellar IMF with $\left(\mathcal{F}_{1}, \Gamma, m_{\max }\right)=(0.12,2.35,100)$ and the Lada \& Lada $(2003)$ distribution of cluster sizes. The solid curve shows the full distribution with $\epsilon_{0}=1 / 3$; the other two distributions use $\epsilon_{0}=1 / 2$ (short dashes) and $\epsilon_{0}=1 / 4$ (long dashes). The vertical lines mark the benchmark flux values for which the background cluster radiation dominates that of the central star (left) and for which the disk is evaporated in $10 \mathrm{Myr}$ (right). b. Same as Figure 9a, but for FUV flux. 
Figure 9 shows the distributions of orbit-averaged fluxes for both the EUV (Fig. 9a) and FUV (Fig. 9b) radiation bands. These distributions are thus the analogs of those shown in Figure 7 (where Fig. 7 was constructed by sampling in radial position, and Fig. 9 was constructed by sampling in energy). In both panels of Figure 9, the solid curve shows the flux distribution with no extinction, the standard stellar IMF, the cluster distribution observed in the solar neighborhood, and the limiting energy value $\epsilon_{0}=1 / 3$. The other two curves show the distributions for other choices of the outer boundary condition: $\epsilon_{0}=1 / 2$ (short-dashes) and $\epsilon_{0}=1 / 4$ (long-dashes). Finally, the two vertical lines are the benchmark values defined in $§ 4.2$. The line on the left corresponds to the flux for which the radiation from the central star is equal to that of the cluster background at a disk radius of $30 \mathrm{AU}$; the line on the right marks the flux for which disks surrounding $1.0 M_{\odot}$ stars are expected to be evaporated over $10 \mathrm{Myr}$ (for assumed disk radius $r_{d}=30 \mathrm{AU}$ and mass $M_{d}=0.05 M_{\odot}$ ). Comparison of the orbit-averaged flux distribution (Fig. 9) with the flux distribution obtained using the distribution of radial positions (Fig. 7) shows that net effect of orbit-averaging makes the flux distribution somewhat narrower and shifts the peak of the distribution to smaller values; the size of the shift is about a factor of $\sim 3$. Note that the difference between the flux distributions shown in Figures 7 and 9 represents an upper limit to the effects of orbit averaging. In real clusters, star-star scattering events and orbital instabilities will act to move stars from one orbit to another, so that they will explore many orbits (many values of $\epsilon$ ). Over sufficiently long times, solar systems will thus experience the full distribution of fluxes depicted in Figure 7.

\section{CONCLUSION}

\subsection{Distributions of UV Radiation Fields}

In this paper we have constructed the distributions of UV radiation fields expected from the observed collection of young embedded groups and clusters. This compilation includes both the EUV and FUV bands. The specification of these radiation fields requires three different types of input distributions: [1] Clusters with a given stellar membership $N$ display a UV luminosity distribution due to sampling of the stellar IMF (note that for $N \lesssim 10^{3}$, the sampling is incomplete and the distributions are wider than their expectation values, as discussed below). [2] The clusters are found over a distribution $f(N)$ of sizes $N$. We consider here both the observed distribution of cluster sizes for the solar neighborhood, where $N$ lies in the range $30 \leq N \leq 2000$, and an extended cluster size distribution where we extrapolate the local distribution up to membership sizes $N=10^{5}$ (Fig. 1). [3] The stars reside at a range of locations within a given cluster, so we must specify the distribution of 
radial positions $P(r)$ in the cluster in order to determine the distribution of radiation flux; alternatively, we can sample the orbital energy distribution function $h(\epsilon)$ and thereby obtain the orbit-averaged radiation fluxes. These probability distributions $\left[f(N), P\left(L_{U V}\right) ; P(r)\right.$ or $h(\epsilon)$ ] jointly determine the composite distribution of fluxes that affect the ensemble of forming solar systems. The first two distributions $\left[f(N), P\left(L_{U V}\right]\right.$ are sufficient to determine the composite distributions of UV luminosities.

Our results show that the distributions of UV radiation are qualitatively different for small clusters and large clusters, where the boundary between the two regimes lies in the range $N=1000-2000$ (note that the boundary is not perfectly sharp). The determination of the radiation fields in the smaller clusters is dominated by incomplete sampling of the stellar IMF, so the resulting distributions are wider than their expectation values and show substantial departures from a gaussian form. In contrast, larger clusters contain enough stars to sample the IMF, so that their UV distributions are close to gaussian, with widths less than their expectation values. According to this criterion, the entire ensemble of clusters in the solar neighborhood falls in the range of "small" clusters, where UV radiation fields are subject to incomplete sampling effects.

The effects of the stellar IMF on the radiation distributions are modest. The upper mass cutoff has little effect, essentially because the IMF is a steeply declining function of stellar mass, so that stars in the mass range $20-40 M_{\odot}$ provide the most UV radiation (Fig. 2). In other words, although more massive stars produce more UV radiation per object, their total contribution is diminished due to their rarity. Shallower slopes for the IMF allow for greater numbers of massive stars and result in a shift in the radiation field distribution to higher values (Fig. 8), whereas steeper IMF slopes act in the opposite direction. The extended cluster distribution produces a composite UV flux distribution that is narrower than that of the solar neighborhood, but retains approximately the same peak value (because the larger cluster radii compensate for larger cluster UV luminosity as $N$ increases).

We have compared the composite flux distributions using both distributions of radial positions (Fig. 7) and distributions of orbital energies (Fig. 9). The first case presents the entire distribution of radiative fluxes provided by the cluster environment. The second case presents the distribution of orbit-averaged fluxes that would be experienced by solar systems as they move through the cluster in the absence of any interactions (which change the orbits). The two distributions are similar, with the orbit-avereged flux distribution being somewhat narrower and shifted to lower values. The statistical measures for the flux distributions shown in Figures $7-9$ are summarized in Table 2 (EUV) and Table 3 (FUV) below. The first column identifies the distribution and gives the corresponding figure and line type in parentheses. The remaining columns specify the mean, median, peak, and 
width of the distributions in terms of the $\log \left(F_{U V}\right)$ values used in the horizontal axis of each corresponding figure.

\subsection{Implications for Star and Planet Formation}

The composite UV flux distributions can be used to provide estimates for the percentages of forming solar systems that have their radiation exposure dominated by the background environment of the cluster (versus the central star). These percentages depend on the mass of the central star and the radius of interest within the disk. For the case of solar type stars and disk radii of $30 \mathrm{AU}$, we find that 42 percent of the population will have their EUV exposure dominated by the background, compared to 58 percent for FUV radiation. Note that the vast majority of the stellar population has mass smaller than $1.0 M_{\odot}$, so the overall percentage of solar systems with radiation dominated by the background will be higher.

Another way to gauge the importance of these background radiation fields is to determine the percentage of disks that will be destroyed by UV radiation before planet formation can take place. For example, we can consider planet formation to be compromised when the disk evaporation time becomes less than $10 \mathrm{Myr}$ at a disk radius of $30 \mathrm{AU}$. For this case, and for the distribution of cluster sizes in the solar neighborhood, we find that 25 percent of the disk population loses some of their planet forming potential due to FUV radiation from the background cluster, whereas only 7 percent of the disk population is compromised by EUV radiation. This latter result is consistent with previous claims (and assumptions) about the relative importance of FUV radiation over EUV radiation in these systems (e.g., Hollenbach et al. 2000; Adams et al. 2004). Again, most stars have smaller masses and their accompanying disks will be more easily destroyed. For red dwarfs with $M_{*}=0.25 M_{\odot}$, for example, 25 percent of the disk population will be evaporated down to a radius of $\sim 8$ $\mathrm{AU}$, which is much smaller than the 30 AU benchmark, and will effectively shut down giant planet formation (see also Laughlin et al. 2004). We note that additional photoevaporation models of evaporating disks must be done to provide further quantification of this issue.

Instead of quantifying the likelihood of disk photoevaporation using the composite flux distribution for the entire cluster sample, we can consider the loss of circumstellar disk gas as a function of stellar membership $N$. This question is vital to future and ongoing searches for extra-solar planets, where clusters are often used as a convenient means of obtaining a well-defined sample of target stars at known distances. The results of this paper show that the radiation fields produced by clusters with smaller $N$ have much more variation from cluster to cluster than their larger $N$ counterparts. However, the mean flux for a cluster is surprisingly insensitive to cluster size $N$. As shown in $\S 4.3$, for sufficiently large $N$ the typical 
Table 2: Measures for EUV Flux Distributions $\left(\log _{10} \mathbf{F}_{E U V}\right)$

\begin{tabular}{lcccc}
\hline \hline Distribution (figure and line type) & Mean & Median & Peak & Width \\
\hline Standard (Fig. 7 - solid) & -1.33 & -0.53 & 0.70 & 2.65 \\
Largest Star Only (Fig. 7 - dotted) & -1.43 & -0.60 & 0.57 & 2.64 \\
Extinction with Q = 3 (Fig. 7 - long dash) & -6.95 & -6.60 & -8.0 & 4.10 \\
Extinction with Q = 10 (Fig. 7 - short dash) & -2.89 & -2.53 & 1.23 & 3.36 \\
IMF [2.1, 100] (Fig. 8 - short dash) & -0.56 & 0.27 & 0.90 & 2.44 \\
IMF [2.5, 100] (Fig. 8 - long dash) & -1.91 & -1.20 & 0.37 & 2.75 \\
IMF [2.35, 120] (Fig. 8 - dot dash) & -1.28 & -0.47 & 0.77 & 2.66 \\
extended cluster (Fig. 8 - dotted) & -0.37 & 0.40 & 0.43 & 2.29 \\
$\epsilon_{0}=1 / 2$ (Fig. 9 - short dash) & -1.79 & -0.93 & 0.37 & 2.62 \\
$\epsilon_{0}=1 / 3$ (Fig. 9 - solid) & -1.98 & -1.13 & 0.10 & 2.62 \\
$\epsilon_{0}=1 / 4$ (Fig. 9 - long dash) & -2.08 & -1.27 & -0.03 & 2.63 \\
\hline \hline
\end{tabular}

Table 3: Measures for FUV Flux Distributions $\left(\log _{10} G_{0}\right)$

\begin{tabular}{lcccc}
\hline \hline Distribution (figure and line type) & Mean & Median & Peak & Width \\
\hline Standard (Fig. 7 - solid) & 3.06 & 3.20 & 3.52 & 1.13 \\
Largest Star Only (Fig. 7 - dotted) & 2.84 & 2.97 & 3.28 & 1.14 \\
Extinction with Q = 3 (Fig. 7 - long dash) & 0.22 & 0.43 & -0.45 & 2.05 \\
Extinction with Q = 10 (Fig. 7 - short dash) & 2.25 & 2.37 & 3.82 & 1.61 \\
IMF [2.1, 100] (Fig. 8 - short dash) & 3.43 & 3.60 & 3.82 & 1.07 \\
IMF [2.5, 100] (Fig. 8 - long dash) & 2.77 & 2.90 & 3.22 & 1.15 \\
IMF [2.35, 120] (Fig. 8 - dot dash) & 3.07 & 3.20 & 3.55 & 1.14 \\
extended cluster (Fig. 8 - dotted) & 3.43 & 3.47 & 3.38 & 0.97 \\
$\epsilon_{0}=1 / 2$ (Fig. 9 - short dash) & 2.58 & 2.77 & 3.22 & 1.05 \\
$\epsilon_{0}=1 / 3$ (Fig. 9 - solid) & 2.38 & 2.57 & 2.98 & 1.06 \\
$\epsilon_{0}=1 / 4$ (Fig. 9 - long dash) & 2.27 & 2.47 & 2.82 & 1.07 \\
\hline \hline
\end{tabular}


UV flux becomes nearly independent of $N$ : In this regime, the total luminosity $L_{U V} \propto N$, the cluster radius scales like $R_{c}^{2} \propto N$, and most of the stars reside in the outer parts of the cluster where $r \sim R_{c}$. As a result, the typical background flux $F_{U V} \propto L_{U V} / r^{2}$ becomes nearly independent of stellar membership size $N$.

Next we note that evaporation tends to remove gas from the outer parts of circumstellar disks, whereas disk accretion drains gaseous material from the inner disk. Taken together, these two processes combine to set the total disk lifetime. As shown herein, disk evaporation from FUV radiations dominates over that of EUV radiation for the expected distributions. Further, the "typical" cluster environment provides enough radiation to evaporate a disk associated with a solar type star down to a radius of $\sim 30 \mathrm{AU}$ in $10 \mathrm{Myr}$. As shown in previous work (e.g., Clarke et al. 2001; Adams et al. 2004), viscous disk accretion with viscosity parameter $\alpha \sim 10^{-3}$ results in a disk lifetime of about 10 Myr for these disk radii $(30 \mathrm{AU})$. As a result, disk lifetimes of this order of magnitude are expected in cluster environments, where, indeed, such disk lifetimes have been observed (Haisch et al. 2001). One prediction of this work is that disks will survive longer in the outer parts of clusters, provided that they do not primarily live on radial orbits. Some observational work on this issue has been carried out, and suggests that the spatial positions of circumstellar disks are anti-correlated with the locations of the massive stars (Guarcello et al. 2007; Balog et al. 2007), but more work along these lines should be done.

EUV leads to ionization (by definition), which has an impact on the efficacy of MRI as a source of disk viscosity. In the absence of any background sources of radiation, beyond the EUV flux from the central star and the background of cosmic rays, circumstellar disks are expected to have "dead zones", regions where the ionization levels are so low that MRI cannot operate (e.g., Gammie 1996). As shown in $\S 4$, when compiled over the observed distribution of clusters in the solar neighborhood, the composite EUV flux distribution shows that 42 percent of the disk population is exposed to significant ionizing radiation from the environment of their birth clusters, in addition to that received from the central stars. This percentage was calculated for a fiducial radius of $30 \mathrm{AU}$; at this location, the vertical extent of the dead zones will be smaller, and the efficacy of MRI and disk accretion will be enhanced for $\sim 40$ percent of the population. At smaller disk radii, however, the percentage of disks that receive a substantial enhancement of ionizing flux from the background cluster is much smaller; specifically, for the background to dominate at $0.1 \mathrm{AU}$, the inner edge of the dead zones, the EUV background flux must be $\sim 10^{5}$ larger than the benchmark value, and the percentage of the distribution with such large flux values is negligible. We thus conclude that the ionizing (EUV) radiation produced by the cluster background is insufficient to eliminate dead zones in circumstellar disks. 
The radiation fields produced by young embedded clusters also have implications for the birth aggregate of our solar system. Since a large fraction of the stellar population is formed within clusters, it is likely that our solar system was born within a cluster of some size $N$. Furthermore, the meteorites from our solar system show evidence of shortlived radioactive isotopes during their early history, and this enrichment is often ascribed to a nearby supernova explosion, which must take place within the birth cluster. Using these observed properties, and others, a number of authors have tried to determine and/or constrain the birth environment of our Sun (e.g., Williams \& Gaidos 2007, Ouellette et al. 2007, Zahnle et al. 2007, Gounelle \& Meibom 2007, Adams \& Laughlin 2001). The radiation fields produced by the cluster environment provide additional constraints: If a hypothetical birth cluster produced too much UV radiation, then the early solar nebula would be evaporated before the giant planets could form. In other words, the existence of our giant planets - in conjunction with disk photoevaporation models - places limits on the environment of our solar system during its first 10 Myr. This paper (Figs. 7,8,9) shows that the expected distribution of clusters provide FUV radiation fields in the range $G_{0}=$ $300-30,000$, with a typical value of $G_{0}=3000$. Over a time span of $10 \mathrm{Myr}$, this latter value of the FUV flux will evaporate a disk around a solar type star down to 36 AU (Adams et al. 2004), thereby leaving enough gas in the solar nebula for giant planets to form. Even a more extreme flux of $G_{0}=30,000$ would only evaporate the disk down to $\sim 15$ AU. As a result, the gas reservoirs for Jupiter and Saturn are always safe, whereas the gas supply for Uranus and Neptune could be compromised (but note that these ice giants have little gas). In addition to photoevaporation, circumstellar disks can be destroyed (or disrupted) in their birth clusters by scattering encounters and by ram pressure stripping (Pfalzner et al. 2006, Olczak et al. 2006, Throop \& Bally 2005, Kobayashi \& Ida 2001). These effects have been included in studies of the solar birth cluster, but can and should be considered more globally (e.g., Adams et al. 2006, Malmberg et al. 2007).

\subsection{Discussion and Future Work}

In addition to continued applications of the distributions of UV radiation fields constructed herein, as outlined above, the distributions themselves can be improved in several ways. The greatest uncertainty concerns the distributions of cluster properties, especially membership size $N$ and radius $R_{c}(N)$. For the solar neighborhood, the recent observational compilations (Lada \& Lada 2003, Porras et al. 2003) provide good working estimates for the distributions of cluster membership size $N$ (see Fig. 1) and cluster radius $R_{c}$ (see eq. 11). Beyond the solar neighborhood, however, no complete observational census has been carried

out, and one must rely on some type of extrapolation. Is this work, we have used an extended 
distribution of the form $d N_{C} / d N \propto N^{-2}$, where $N_{C}$ is the number of clusters, so that equal numbers of stars are formed within each decade of cluster size $N$ [i.e., $N\left(d N_{C} / d N\right) \propto N^{-1}$ ]. For clusters with large $N$, the sampling of the stellar IMF is relatively complete, so that the statistics of the distribution of luminosity are well-behaved. In order to determine the distribution of UV fluxes, one needs the distribution of radial positions within the cluster, including the cluster radius $R_{c}$. Since most of the cluster members do not live in the cluster core, but rather in its outer parts, the distribution of radial sizes is crucial. In the present formulation, the adopted relation $R_{c} \propto N^{1 / 2}$ (observed in the solar neighborhood) implies that although clusters with larger $N$ have correspondingly larger luminosities, they produce almost the same distribution of fluxes because of their larger radii. On the other hand, if clusters with larger $N$ do not follow this empirical law (e.g., they could be more compact), then the distributions of UV fluxes would be shifted toward higher values. Another possibility is that the population of larger clusters has mean radii given by equation (1), but the radii sample a wide distribution about the mean, so that the more compact clusters would produce environments with large UV fluxes.

Another related issue is that this work samples the stellar IMF independently for clusters of all sizes $N$. Current observartions are consistent with the assumption that the IMF is independent of environment (e.g., Kroupa 2002, Chabrier 2003, and references therein). However, correlations of stellar IMF with cluster size $N$ could affect the distributions of UV radiation fields calculated here. Additional observation work should be carried out to determine if any such correlations exist, and how they are quantified.

In addition to EUV and FUV radiation, young stars also produce copious amounts

of X-ray emission. As a result, cluster environments can also provide a significant X-ray background radiation field, which can also affect star and planet formation. In this case, the dominant effect is ionization, both in circumstellar disks and in pre-stellar cores. In the case of disks, ionization helps MRI produce disk accretion, and thereby helps the star formation process. In pre-stellar cores, ionization leads to greater coupling between gas the magnetic fields, and thereby slows down star formation. In future work, the construction of $\mathrm{X}$-ray backgrounds in clusters, and the effects of this radiation on star formation, should be considered.

\section{Acknowledgment}

We thank Lori Allen, Tom Megeath, Phil Myers, and Eva Proszkow for useful discussions an an anonymous referee for useful comments that improved the paper. This work was supported at the University of Michigan by the Michigan Center for Theoretical Physics, by the Astrophysics Theory Program (NNG04GK56G0), and by the Spitzer Space Telescope 
Theoretical Research Program (1290776). This work was supported at Xavier University through the Hauck Foundation.

\section{REFERENCES}

Adams, F. C., \& Bloch, A. M. 2005, ApJ, 629, 204 (AB05)

Adams, F. C., \& Fatuzzo, M. 1996, ApJ, 464, 256

Adams, F. C., Hollenbach, D., Laughlin, G., \& Gorti, U. 2004, ApJ, 611, 360

Adams, F. C., \& Laughlin, G. 2001, Icarus, 150, 151

Adams, F. C., \& Myers, P. C. 2001, ApJ, 553, 744

Adams, F. C., Proszkow, E. M., Fatuzzo, M., \& Myers, P. C. 2006, ApJ, 641, 504 (APFM)

Alexander, R. D., Clarke, C. J., \& Pringle, J. E. 2006, MNRAS, 369, 229

Allen, L. E., Megeath, S. T., Gutermuth, R., Myers, P. C., Adams, F. C., Muzzerolle, J., Young, E., \& Pipher, J. L. 2007, Protostars and Planets V, ed. B. Reipurth (Tucson: Univ. Ariz. Press), p. 361

Armitage, P. J. 2000, A\&A, 362, 968

Balbus, S., \& Hawley, J. 1991, ApJ, 376, 214

Balog, Z, Muzerolle, J., Rieke, G. H., Su, K. Y. L, \& Young, E. T. 2007, ApJ, 660, 1532

Bethell, T. J., Zweibel, E. G., \& Li, P. S. 2007, ApJ, 667, 275

Binney, J., \& Tremaine, S. 1987, Galactic Dynamics, (Princeton: Princeton Univ. Press)

Carpenter, J. M. 2000, AJ, 120, 3139

Chabrier, G. 2003, PASP, 115, 763

Clarke, C. J., Gendrin, A., Sotomayor, M. 2001, MNRAS, 328, 485

Elmegreen, B. G., Efremov, Y., Pudritz, R. E., \& Zinnecker, H. 2000, Protostars and Planets IV, eds. V. Mannings, A. Boss, \& S. Russell (Tucson: Univ. Ariz. Press), p. 179

Gammie, C. F. 1996, ApJ, 457, 355 
Guarcello, M. G., Prisinzano, L., Micela, G., Damiani, F., Peres, G., \& Sciortino, S. 2007, A\&A, 462, 245

Gorti, U., \& Hollenbach, D. 2002, ApJ, 573, 215

Gounelle, M., \& Meibom, A. 2007, submitted to ApJ

Haisch, K. E., Lada, E. A., \& Lada, C. J. 2001, ApJ, 553, L153

Hernquist, L. 1990, ApJ, 356, 359

Hollenbach, D., Johnstone, D., Lizano, S., \& Shu, F. 1994, ApJ, 654, 669

Hollenbach, D. J., Yorke, H. W., \& Johnstone, D. 2000, Protostars and Planets IV, eds. V. Mannings, A. Boss, \& S, Russell (Tucson: Univ. Ariz. Press), p. 401

Kobayashi, H., \& Ida, S. 2001, Icarus, 153, 416

Kroupa, P. 2002, Science, 295, 82

Lada, C. J., \& Lada, E. A. 2003, ARA\&A, 41, 57

Laughlin, G., Bodenheimer, P., \& Adams, F. C. 2004, ApJ, 612, 73

Maeder, A., \& Meynet, G. 1987, A\&A, 182, 243

Malmberg, D., de Angeli, F., Davies, M. B., Church, R. P., Mackey, D., \& Wilkinson, M. I. 2007, MNRAS, 378, 1207

Massey, P. 2003, ARA\&A, 41, 15

Olczak, C., Pfalzner, S., \& Spurzem, R. 2006, ApJ, 642, 1140

Ouellette, N., Desch, S. J., Hester, J. J. 2007, ApJ, 662, 12680

Parravano, A., Hollenbach, D. J., \& McKee, C. F. 2003, ApJ, 584, 797

Pfalzner, S., Olczak, C., \& Eckart, A. 2006, A\&A, 454, 811

Porras, A., et al. 2003, AJ, 126, 1916

Richtmyer, R. D. 1978, Principles of Advanced Mathematical Physics (New York: Springer)

Salpeter, E. E. 1955, ApJ, 121, 161

Scally, A., \& Clarke, C. 2001, MNRAS, 325, 449 
Schaller, G., Schaerer, D., Meynet, G., \& Maeder, A. 1992, A\&AS, 96, 269

Shu, F. H. 1992, Gas Dynamics, (Mill Valley: Univ. Sci. Books)

Shu, F. H., Adams, F. C., \& Lizano, S. 1987, ARA\&A, 25, 23

Shu, F. H., Johnstone, D., \& Hollenbach, D. J. 1993, Icarus, 106, 92

Störzer, H., \& Hollenbach, D. 1999, ApJ, 515, 688

Testi, L., Palla, F., \& Natta, A. 1998, A\&AS, 133, 81

Throop, H. B., \& Bally, J. 2005, ApJ, 623, 149

Williams, J. P., \& Gaidos, E. 2007, ApJ, 663, 33

Zahnle, K., Arndt, N., Cockell, C., Halliday, A., Nisbet, E., Selsis, F., \& Sleep, N. H. 2007, SSRv, 129, 35Z

This preprint was prepared with the AAS IATEX macros v5.2. 